\title{
Affinoids in the Lubin-Tate perfectoid space and simple supercuspidal representations II: wild case
}

\author{
Naoki Imai ${ }^{1} \mathbb{D} \cdot$ Takahiro Tsushima $^{2}$
}

Received: 13 April 2020 / Revised: 13 April 2020 / Accepted: 17 October 2020 / Published online: 9 January 2021 (C) The Author(s) 2020

\begin{abstract}
We construct a family of affinoids in the Lubin-Tate perfectoid space and their formal models such that the middle cohomology of their reductions realizes the local Langlands correspondence and the local Jacquet-Langlands correspondence for the simple supercuspidal representations. The reductions of the formal models are isomorphic to the perfections of some Artin-Schreier varieties, whose cohomology realizes primitive Galois representations. We show also the Tate conjecture for Artin-Schreier varieties associated to quadratic forms.
\end{abstract}

\section{Introduction}

Let $K$ be a non-archimedean local field with residue field $k$. Let $p$ be the characteristic of $k$. We write $\mathcal{O}_{K}$ for the ring of integers of $K$, and $\mathfrak{p}$ for the maximal ideal of $\mathcal{O}_{K}$. We fix an algebraic closure $k^{\mathrm{ac}}$ of $k$. The Lubin-Tate spaces are deformation spaces of the one-dimensional formal $\mathcal{O}_{K}$-module over $k^{\text {ac }}$ of height $n$ with level structures. We take a prime number $\ell$ that is different from $p$. The local Langlands correspondence (LLC) and the local Jacquet-Langlands correspondence (LJLC) for supercuspidal representations of $G L_{n}$ are realized in the $\ell$-adic cohomology of Lubin-Tate spaces. This is proved in [4] and [9] by global automorphic arguments. On the other hand, the

\section{Communicated by Wei Zhang.}

Naoki Imai

naoki@ms.u-tokyo.ac.jp

Takahiro Tsushima

tsushima@math.s.chiba-u.ac.jp

1 Graduate School of Mathematical Sciences, The University of Tokyo, 3-8-1 Komaba, Meguro-ku, Tokyo 153-8914, Japan

2 Department of Mathematics and Informatics, Faculty of Science, Chiba University, 1-33 Yayoi-cho, Inage, Chiba 263-8522, Japan 
relation between these correspondences and the geometry of Lubin-Tate spaces is not well understood.

In this direction, Yoshida constructs a semi-stable model of the Lubin-Tate space with a full level $\mathfrak{p}$-structure, and studies its relation with the LLC in [27]. In this case, the Deligne-Lusztig varieties appear as open subschemes in the reductions of the semi-stable models, and their cohomology realizes the LLC for depth zero supercuspidal representations. In [5], Boyarchenko-Weinstein construct a family of affinoids in the Lubin-Tate perfectoid space and their formal models so that the cohomology of the reductions realizes the LLC and the LJLC for some representations which are related to unramified extensions of $K$ ( $c f$. [25] for some special case at a finite level). It generalizes a part of the result in [27] to higher conductor cases. In the Lubin-Tate perfectoid setting, the authors study the case for the essentially tame simple supercuspidal representations in [12], where simple supercuspidal means that the exponential Swan conductor is equal to one. See [1] for the notion of essentially tame representations. The result in [12] is generalized to some higher conductor essentially tame cases by Tokimoto in [24] ( $c f$. [14] for some special case at a finite level).

In all the above cases, Langlands parameters are of the form $\operatorname{Ind}_{W_{L}}^{W_{K}} \chi$ for a finite separable extension $L$ over $K$ and a character $\chi$ of $W_{L}$, where $W_{K}$ and $W_{L}$ denote the Weil groups of $K$ and $L$ respectively. Further, the construction of affinoids directly involves $C M$ points which have multiplication by $L$. In this paper, we study the case for simple supercuspidal representations which are not essentially tame. In this case, the Langlands parameters can not be written as inductions of characters. Hence, we have no canonical candidate of $\mathrm{CM}$ points which may be used for constructions of affinoids.

We will explain our main result. All the representation are essentially tame if $n$ is prime to $p$. Hence, we assume that $p$ divides $n$. We say that a representation of $G L_{n}(K)$ is essentially simple supercuspidal if it is a character twist of a simple supercuspidal representation. Let $q$ be the number of the elements of $k$ and $D$ be the central division algebra over $K$ of invariant $1 / n$. We write $q=p^{f}$ and $n=p^{e} n^{\prime}$, where $n^{\prime}$ is prime to $p$. We put $m=\operatorname{gcd}(e, f)$. The main theorem is the following:

Theorem For $r \in \mu_{q-1}(K)$, there is an affinoid $\mathcal{X}_{r}$ in the Lubin-Tate perfectoid space and its formal model $\mathfrak{X}_{r}$ such that

- the special fiber $\overline{\mathfrak{X}}_{r}$ of $\mathfrak{X}_{r}$ is isomorphic to the perfection of the affine smooth variety defined by

$$
z^{p^{m}}-z=y^{p^{e}+1}-\frac{1}{n^{\prime}} \sum_{1 \leq i \leq j \leq n-2} y_{i} y_{j} \text { in } \mathbb{A}_{k^{\mathrm{ac}}}^{n}
$$

- the stabilizer $H_{r} \subset G L_{n}(K) \times D^{\times} \times W_{K}$ of $\mathcal{X}_{r}$ naturally acts on $\overline{\mathfrak{X}}_{r}$, and - c-Ind $H_{r}^{G L_{n}(K) \times D^{\times} \times W_{K}} H_{c}^{n-1}\left(\overline{\mathfrak{X}}_{r}, \overline{\mathbb{Q}}_{\ell}\right)$ realizes the LLC and the LJLC for essentially simple supercuspidal representations.

See Theorem 2.6 and Theorem 6.5 for precise statements. As we mentioned, we have no candidate of $\mathrm{CM}$ points for the construction of affinoids. First, we consider 
a CM point $\xi$ which has multiplication by a field extension of $K$ obtained by adding an $n$-th root of a uniformizer of $K$. If we imitate the construction of affinoids in [12] using the CM point $\xi$, we can get a non-trivial affinoid and its model, but the reduction degenerates in some sense, and the cohomology of the reduction does not give a supercuspidal representation. What we will do in this paper is to modify the CM point $\xi$ using information of field extensions which appear in the study of our simple supercuspidal Langlands parameter. The modified point, which is constructed in Proposition 2.2, is no longer a CM point, but we can use this point for a construction of a desired affinoid. Since the modification comes from the study of the Langlands parameter, we expect that such constructions work also for other Langlands parameters.

In the above mentioned preceding researches, the Langlands parameters are inductions of characters, and realized from commutative group actions on varieties. In the case for Deligne-Lusztig varieties, they come from the natural action of tori. In our simple supercuspidal case, they come from non-commutative group actions. For example, the restriction to the inertia subgroup of a simple supercuspidal Langlands parameter factors through a semidirect product of a cyclic group with a Heisenberg type group, which acts on our Artin-Schreier variety in a very non-trivial way.

In the following, we briefly explain the content of each section. In Sect. 1, we collect known results on the Lubin-Tate perfectoid space, its formal model and group action on it.

In Sect. 2, we construct a family of affinoids and their formal models. Further we determine the reductions of them. The reduction is isomorphic to the perfection of some Artin-Schreier variety.

In Sect. 3, we describe the group action on the reductions. In Sect. 4, we show that the Tate conjecture holds for Artin-Schreier varieties of associated to quadratic forms. Further, we study the action of some special element on cycle classes in the etale cohomology of the Artin-Schreier variety. This becomes a key ingredient for the proof of the main theorem.

In Sect. 5, we give an explicit description of the LLC and the LJLC for essentially simple supercuspidal representations, which follows from results in [13] and [17]. In Sect. 6, we give a geometric realization of the LLC and the LJLC in the cohomology of our reduction.

\section{Notation}

For a non-archimedean valuation field $F$, its valuation ring is denoted by $\mathcal{O}_{F}$. For a non-archimedean valuation field $F$ and an element $a \in \mathcal{O}_{F}$, its image in the residue field is denoted by $\bar{a}$. For $a \in \mathbb{Q}$ and elements $f, g$ with valuation $v$ that takes values in $\mathbb{Q}$, we write $f \equiv g \bmod _{\geq} a$ if $v(f-g) \geq a$, and $f \equiv g \bmod _{>} a$ if $v(f-g)>a$. For a topological field extension $E$ over $F$, let $\operatorname{Gal}(E / F)$ denote the group of the continuous automorphisms of $E$ over $F$. For an ideal $I$ of a topological ring, let $I^{-}$ denote the closure of $I$. 


\section{Lubin-Tate perfectoid space}

\subsection{Lubin-Tate perfectoid space and its formal model}

Let $K$ be a non-archimedean local field with residue field $k$ of characteristic $p$. Let $q$ be the number of the elements of $k$. We write $\mathfrak{p}$ for the maximal ideal of $\mathcal{O}_{K}$. We fix an algebraic closure $K^{\text {ac }}$ of $K$. Let $k^{\text {ac }}$ be the residue field of $K^{\text {ac }}$.

Let $n$ be a positive integer. We take a one-dimensional formal $\mathcal{O}_{K}$-module $\mathcal{G}_{0}$ over $k^{\text {ac }}$ of height $n$, which is unique up to isomorphism. Let $K^{\text {ur }}$ be the maximal unramified extension of $K$ in $K^{\text {ac }}$. We write $\widehat{K}^{\text {ur }}$ for the completion of $K^{\text {ur }}$. Let $\left\{\text { Spf } A_{m}\right\}_{m \geq 0}$ be the tower of Lubin-Tate formal schemes defined by Drinfeld level $\mathfrak{p}^{m}$-structure as explained in $[12, \S 1.1]$. Note that the generic fibers of these formal schemes are connected components of usual Lubin-Tate spaces. Let $I$ the ideal of $\lim _{m} A_{m}$ generated by the maximal ideal of $A_{0}$. Let $A$ be the $I$-adic completion of $\overleftrightarrow{\lim _{m}} A_{m}$. We put $\mathbf{M}_{\mathcal{G}_{0}, \infty}=\operatorname{Spf} A$.

Let $K^{\text {ab }}$ be the maximal abelian extension of $K$ in $K^{\text {ac }}$. We write $\widehat{K}^{\text {ab }}$ for the completion of $K^{\mathrm{ab}}$. Let $\wedge \mathcal{G}_{0}$ denote the one-dimensional formal $\mathcal{O}_{K}$-module over $k^{\text {ac }}$ of height one. Then we have $\mathbf{M}_{\wedge \mathcal{G}_{0}, \infty} \simeq \operatorname{Spf} \mathcal{O}_{\widehat{K}}$ ab by the Lubin-Tate theory. We have a determinant morphism

$$
\mathbf{M}_{\mathcal{G}_{0}, \infty} \rightarrow \mathbf{M}_{\wedge \mathcal{G}_{0}, \infty}
$$

by [26, 2.5 and 2.7] (cf. [8]). Then, we have the ring homomorphism $\mathcal{O}_{\widehat{K}}$ ab $\rightarrow A$ determined by (1.1).

We fix a uniformizer $\varpi$ of $K$. Let $\mathcal{M}_{\infty}$ be the open adic subspace of $\operatorname{Spa}(A, A)$ defined by $|\varpi(x)| \neq 0(c f$. $[10,2])$. We regard $\mathcal{M}_{\infty}$ as an adic space over $\widehat{K}^{\text {ur }}$. Let $\mathbf{C}$ be the completion of $K^{\text {ac }}$. For a deformation $\mathcal{G}$ of $\mathcal{G}_{0}$ over $\mathcal{O}_{\mathbf{C}}$, we put

$$
V_{\mathfrak{p}}(\mathcal{G})=\left(\lim _{\longleftarrow} \mathcal{G}\left(\mathcal{O}_{\mathbf{C}}\right)\left[\mathfrak{p}^{m}\right]\right) \otimes_{\mathcal{O}_{K}} K
$$

where $\mathcal{G}\left(\mathcal{O}_{\mathbf{C}}\right)\left[\mathfrak{p}^{m}\right]$ denotes the $\mathcal{O}_{K}$-module of the $\mathfrak{p}^{m}$-torsion points of $\mathcal{G}\left(\mathcal{O}_{\mathbf{C}}\right)$ and the transition maps are multiplications by $\varpi$. By the construction, each point of $\mathcal{M}_{\infty}(\mathbf{C})$ corresponds to a triple $(\mathcal{G}, \phi, \iota)$ that consists of a formal $\mathcal{O}_{K}$-module $\mathcal{G}$ over $\mathcal{O}_{\mathbf{C}}$, an isomorphism $\phi: K^{n} \rightarrow V_{\mathfrak{p}}(\mathcal{G})$ and an isomorphism $\iota: \mathcal{G}_{0} \rightarrow \mathcal{G} \otimes_{\mathcal{O}_{\mathbf{C}}} k^{\text {ac }}(c f .[5$, Definition 2.10.1]).

We put $\eta=\operatorname{Spa}\left(\widehat{K}^{\mathrm{ab}}, \mathcal{O}_{\widehat{K}^{\mathrm{ab}}}\right)$. By the ring homomorphism $\mathcal{O}_{\widehat{K}^{\mathrm{ab}}} \rightarrow A$, we can regard $\mathcal{M}_{\infty}$ as an adic space over $\eta$, for which we write $\mathcal{M}_{\infty, \eta}$. We put $\bar{\eta}=\operatorname{Spa}\left(\mathbf{C}, \mathcal{O}_{\mathbf{C}}\right)$ and $\mathcal{M}_{\infty, \bar{\eta}}=\mathcal{M}_{\infty, \eta} \times_{\eta} \bar{\eta}$. Then, $\mathcal{M}_{\infty, \bar{\eta}}$ is a perfectoid space over $\mathbf{C}$ in the sense of [21, Definition 6.15] by [26, Lemma 2.32]. We call $\mathcal{M}_{\infty, \bar{\eta}}$ the Lubin-Tate perfectoid space.

In the following, we recall an explicit description of $A^{\circ}=A \widehat{\otimes}_{\mathcal{O}_{\widehat{K}} \text { ab }} \mathcal{O}_{\mathbf{C}}$ given in $[26,(2.8)]$. Let $\widehat{\mathcal{G}_{0}}$ be the formal $\mathcal{O}_{K}$-module over $\mathcal{O}_{K}$ whose logarithm is

$$
\sum_{i=0}^{\infty} \frac{X^{q^{i n}}}{\varpi^{i}}
$$


(cf. [5, 2.3]). Let $\mathcal{G}_{0}$ be the formal $\mathcal{O}_{K}$-module over $k^{\text {ac }}$ obtained as the reduction of $\widehat{\mathcal{G}_{0}}$. We put $\mathcal{O}_{D}=$ End $\mathcal{G}_{0}$ and $D=\mathcal{O}_{D} \otimes_{\mathcal{O}_{K}} K$, which is the central division algebra over $K$ of invariant $1 / n$. Let $[\cdot]$ denote the action of $\mathcal{O}_{D}$ on $\mathcal{G}_{0}$. Let $\varphi$ be the element of $D$ such that $[\varphi](X)=X^{q}$. Let $K_{n}$ be the unramified extension of $K$ of degree $n$. We consider the $K$-algebra embedding of $K_{n}$ into $D$ determined by

$$
[\zeta](X)=\bar{\zeta} X \text { for } \zeta \in \mu_{q^{n}-1}\left(K_{n}\right)
$$

Then we have $\varphi^{n}=\varpi$ and $\varphi \zeta=\zeta^{q} \varphi$ for $\zeta \in \mu_{q^{n}-1}\left(K_{n}\right)$. Let $\widehat{\wedge \mathcal{G}_{0}}$ be the onedimensional formal $\mathcal{O}_{K}$-module over $\mathcal{O}_{K}$ whose logarithm is

$$
\sum_{i=0}^{\infty}(-1)^{(n-1) i} \frac{X^{q^{i}}}{\varpi^{i}}
$$

We choose a compatible system $\left\{t_{m}\right\}_{m \geq 1}$ such that

$$
t_{m} \in K^{\mathrm{ac}} \quad(m \geq 1), \quad t_{1} \neq 0, \quad[\varpi]_{\wedge \mathcal{G}_{0}}\left(t_{1}\right)=0, \quad[\varpi]_{\wedge \mathcal{G}_{0}}\left(t_{m}\right)=t_{m-1} \quad(m \geq 2)
$$

We put

$$
t=\lim _{m \rightarrow \infty}(-1)^{q(n-1)(m-1)} t_{m}^{q^{m-1}} \in \mathcal{O}_{\mathbf{C}}
$$

Let $v$ be the normalized valuation of $K$ such that $v(\varpi)=1$. The valuation $v$ naturally extends to a valuation on $\mathbf{C}$, for which we again write $v$. Note that $v(t)=1 /(q-1)$. For an integer $i \geq 0$, we put

$$
t^{q^{-i}}=\lim _{m \rightarrow \infty}(-1)^{q(n-1)(m-1)} t_{m}^{q^{m-i-1}} .
$$

Let $W_{K}$ be the Weil group of $K$. Let $\operatorname{Art}_{K}: K^{\times} \stackrel{\sim}{\rightarrow} W_{K}^{\text {ab }}$ be the Artin reciprocity map normalized such that a uniformizer is sent to a lift of the geometric Frobenius element. We use similar normalizations also for the Artin reciprocity maps for other non-archimedean local fields. Let $\sigma \in W_{K}$. Let $n_{\sigma}$ be the image of $\sigma$ under the composite

$$
W_{K} \rightarrow W_{K}^{\mathrm{ab}} \stackrel{\mathrm{Art}_{K}^{-1}}{\longrightarrow} K^{\times} \stackrel{v}{\rightarrow} \mathbb{Z}
$$

Let $a_{K}: W_{K} \rightarrow \mathcal{O}_{K}^{\times}$be the homomorphism given by the action of $W_{K}$ on $\left\{t_{m}\right\}_{m \geq 1}$. It induces an isomorphism $a_{K}: \operatorname{Gal}\left(\widehat{K}^{\mathrm{ab}} / \widehat{K}^{\mathrm{ur}}\right) \simeq \mathcal{O}_{K}^{\times}$.

For $m \geq 0$, we put

$$
\delta_{m}\left(X_{1}, \ldots, X_{n}\right)=\widehat{\wedge \mathcal{G}_{0}} \sum_{\left(m_{1}, \ldots, m_{n}\right)} \operatorname{sgn}\left(m_{1}, \ldots, m_{n}\right) X_{1}^{q^{m_{1}-m}} \cdots X_{n}^{q^{m_{n}-m}}
$$


in $\mathcal{O}_{K}\left[\left[X_{1}^{1 / q^{\infty}}, \ldots, X_{n}^{1 / q^{\infty}}\right]\right]$, where

- the symbol $\widehat{\wedge \mathcal{G}_{0}} \sum$ denotes the sum under the additive operation of $\widehat{\wedge \mathcal{G}_{0}}$,

- we take the sum over $n$-tuples $\left(m_{1}, \ldots, m_{n}\right)$ of integers such that $m_{1}+\cdots+m_{n}=$ $n(n-1) / 2$ and $m_{i} \not \equiv m_{j} \bmod n$ for $i \neq j$,

- $\operatorname{sgn}\left(m_{1}, \ldots, m_{n}\right)$ is the sign of the permutation on $\mathbb{Z} / n \mathbb{Z}$ defined by $i \mapsto m_{i+1}$.

We put

$$
\delta=\lim _{m \rightarrow \infty} \delta_{m}^{q^{m}} \in \mathcal{O}_{\mathbf{C}}\left[\left[X_{1}^{1 / q^{\infty}}, \ldots, X_{n}^{1 / q^{\infty}}\right]\right]
$$

For $l \geq 1$, we put

$$
\delta^{q^{-l}}=\lim _{m \rightarrow \infty} \delta_{m}^{q^{m-l}}
$$

The following theorem follows from [26, (2.8)] and the proof of [5, Theorem 2.10.3] (cf. [23, Theorem 6.4.1]).

Theorem 1.1 ([12, Theorem 1.3]) Let $\sigma \in \operatorname{Gal}\left(\widehat{K}^{\mathrm{ab}} / \widehat{K}^{\mathrm{ur}}\right)$. We put $A^{\sigma}=A \widehat{\otimes}_{\mathcal{O}_{\widehat{K}}^{\mathrm{ab}}, \sigma} \mathcal{O}_{\mathbf{C}}$. Then, we have an isomorphism

$$
A^{\sigma} \simeq \mathcal{O}_{\mathbf{C}}\left[\left[X_{1}^{1 / q^{\infty}}, \ldots, X_{n}^{1 / q^{\infty}}\right]\right] /\left(\delta\left(X_{1}, \ldots, X_{n}\right)^{q^{-m}}-\sigma\left(t^{q^{-m}}\right)\right)_{m \geq 0}^{-} .
$$

For $\sigma \in \operatorname{Gal}\left(\widehat{K}^{\mathrm{ab}} / \widehat{K}^{\text {ur }}\right)$, let $\mathcal{M}_{\infty, \bar{\eta}, \sigma}$ be the base change of $\mathcal{M}_{\infty, \eta}$ by $\bar{\eta} \rightarrow \eta \stackrel{\sigma}{\rightarrow} \eta$. For $\sigma \in \operatorname{Gal}\left(\widehat{K}^{\mathrm{ab}} / \widehat{K}^{\mathrm{ur}}\right)$ and $\alpha=a_{K}(\sigma) \in \mathcal{O}_{K}^{\times}$, we write $A^{\alpha}$ for $A^{\sigma}$ and $\mathcal{M}_{\infty, \bar{\eta}, \alpha}^{(0)}$ for $\mathcal{M}_{\infty, \bar{\eta}, \sigma}^{(0)}$. We put

$$
\mathbf{M}_{\infty, \mathcal{O}_{\mathbf{C}}}^{(0)}=\coprod_{\alpha \in \mathcal{O}_{K}^{\times}} \operatorname{Spf} A^{\alpha}, \quad \mathcal{M}_{\infty, \bar{\eta}}^{(0)}=\coprod_{\alpha \in \mathcal{O}_{K}^{\times}} \mathcal{M}_{\infty, \bar{\eta}, \alpha} .
$$

Then $\mathcal{M}_{\infty, \bar{\eta}}^{(0)}$ is the generic fiber of $\mathbf{M}_{\infty, \mathcal{O}_{\mathbf{C}}}^{(0)}$, and $\mathcal{M}_{\infty, \bar{\eta}}^{(0)}(\mathbf{C})=\mathcal{M}_{\infty}(\mathbf{C})$.

Let $+\widehat{\mathcal{G}_{0}}$ and $+\widehat{\widehat{\wedge \mathcal{G}_{0}}}$ be the additive operations for $\widehat{\mathcal{G}_{0}}$ and $\widehat{\wedge \mathcal{G}_{0}}$ respectively.

Lemma 1.2 ([12, Lemma 1.5])

(1) We have $X_{1}+\widehat{\mathcal{G}_{0}} X_{2} \equiv X_{1}+X_{2}$ modulo terms of total degree $q^{n}$.

(2) We have $X_{1}+\frac{\widehat{\mathcal{G}}_{0}}{\mathcal{G}_{0}} X_{2} \equiv X_{1}+X_{2}$ modulo terms of total degree $q$.

Let $\boldsymbol{X}_{i}$ be $\left(X_{i}^{q^{-j}}\right)_{j \geq 0}$ for $1 \leq i \leq n$. We write $\delta\left(\boldsymbol{X}_{1}, \ldots, \boldsymbol{X}_{n}\right)$ for the $q$-th power compatible system $\left(\delta\left(X_{1}, \ldots, X_{n}\right)^{q^{-j}}\right)_{j \geq 0}$.

For $q$-th power compatible systems $\boldsymbol{X}=\left(X^{q^{-j}}\right)_{j \geq 0}$ and $\boldsymbol{Y}=\left(Y^{q^{-j}}\right)_{j \geq 0}$ that take values in $\mathcal{O}_{\mathbf{C}}$, we define $q$-th power compatible systems $\boldsymbol{X}+\boldsymbol{Y}, \boldsymbol{X}-\boldsymbol{Y}$ and $\boldsymbol{X} \boldsymbol{Y}$ by the requirement that their $j$-th components for $j \geq 0$ are

$$
\lim _{m \rightarrow \infty}\left(X^{q^{-m}}+Y^{q^{-m}}\right)^{q^{m-j}}, \quad \lim _{m \rightarrow \infty}\left(X^{q^{-m}}-Y^{q^{-m}}\right)^{q^{m-j}}, \text { and } X^{q^{-j}} Y^{q^{-j}}
$$


respectively. For such $\boldsymbol{X}=\left(X^{q^{-j}}\right)_{j \geq 0}$, we put $v(\boldsymbol{X})=v(X)$. We put

$$
\delta_{0}^{\prime}\left(\boldsymbol{X}_{1}, \ldots, \boldsymbol{X}_{n}\right)=\sum_{\left(m_{1}, \ldots, m_{n}\right)} \operatorname{sgn}\left(m_{1}, \ldots, m_{n}\right) \boldsymbol{X}_{1}^{q^{m_{1}}} \cdots \boldsymbol{X}_{n}^{q^{m_{n}}}
$$

where we take the sum in the above sense and the index set is the same as (1.3).

Lemma 1.3 ([12, Lemma 1.6])] Assume that $n \geq 2$ and $v\left(\boldsymbol{X}_{i}\right) \geq\left(n q^{i-1}(q-1)\right)^{-1}$ for $1 \leq i \leq n$. Then, we have

$$
\delta\left(\boldsymbol{X}_{1}, \ldots, \boldsymbol{X}_{n}\right) \equiv \delta_{0}^{\prime}\left(\boldsymbol{X}_{1}, \ldots, \boldsymbol{X}_{n}\right) \quad \bmod _{>} \frac{1}{n}+\frac{1}{q-1}
$$

\subsection{Group action on the formal model}

We define a group action on the formal scheme $\mathbf{M}_{\infty, \mathcal{O}_{\mathbf{C}}}^{(0)}$, which is compatible with usual group actions on Lubin-Tate spaces with finite level ( $c f .[5,2.11])$. We put

$$
G=G L_{n}(K) \times D^{\times} \times W_{K}
$$

Let $G^{0}$ denote the kernel of the following homomorphism:

$$
G \rightarrow \mathbb{Z} ; \quad(g, d, \sigma) \mapsto v\left(\operatorname{det}(g)^{-1} \operatorname{Nrd}_{D / K}(d) \operatorname{Art}_{K}^{-1}(\sigma)\right) .
$$

Then, the formal scheme $\mathbf{M}_{\infty, \mathcal{O}_{\mathrm{C}}}^{(0)}$ admits a right action of $G^{0}$. We write down the action. In the sequel, we use the following notation:

For $a \in \mu_{q^{n-1}}\left(K_{n}\right) \cup\{0\}$, let $a^{q^{-m}}$ denote the $q^{m}$-th root of $a$ in $\mu_{q^{n}-1}\left(K_{n}\right) \cup\{0\}$ for a positive integer $m$, and we simply write $a$ also for the $q$-th power compatible system $\left(a^{q^{-m}}\right)_{m \geq 0}$.

For $q$-th power compatible systems $\boldsymbol{X}=\left(X^{q^{-j}}\right)_{j \geq 0}$ and $\boldsymbol{Y}=\left(Y^{q^{-j}}\right)_{j \geq 0}$ that take values in $\mathcal{O}_{\mathbf{C}}$, we define a $q$-th power compatible system $\boldsymbol{X}+\widehat{\mathcal{G}_{0}} \boldsymbol{Y}$ by the requirement that their $j$-th components for $j \geq 0$ are

$$
\lim _{m \rightarrow \infty}\left(X^{q^{-m}}+\widehat{\mathcal{G}_{0}} Y^{q^{-m}}\right)^{q^{m-j}}
$$

The symbol $\widehat{\mathcal{G}_{0}} \sum$ denotes this summation for $q$-th power compatible systems.

First, we define a left action of $G L_{n}(K) \times D^{\times}$on the ring

$$
B_{n}=\mathcal{O}_{\mathbf{C}}\left[\left[X_{1}^{1 / q^{\infty}}, \ldots, X_{n}^{1 / q^{\infty}}\right]\right] .
$$

For $a=\sum_{j=l}^{\infty} a_{j} \varpi^{j} \in K$ with $l \in \mathbb{Z}$ and $a_{j} \in \mu_{q-1}(K) \cup\{0\}$, we set

$$
[a] \cdot \boldsymbol{X}_{i}=\widehat{\mathcal{G}_{0}} \sum_{j=l}^{\infty} a_{j} \boldsymbol{X}_{i}^{q^{j n}}
$$


for $1 \leq i \leq n$. Let $g \in G L_{n}(K)$. We write $g=\left(a_{i, j}\right)_{1 \leq i, j \leq n}$. Then, let $g$ act on the ring $B_{n}$ by

$$
g^{*}: B_{n} \rightarrow B_{n} ; \boldsymbol{X}_{i} \mapsto \widehat{\mathcal{G}_{0}} \sum_{j=1}^{n}\left[a_{j, i}\right] \cdot \boldsymbol{X}_{j} \quad \text { for } 1 \leq i \leq n
$$

Let $d \in D^{\times}$. We write $d^{-1}=\sum_{j=l}^{\infty} d_{j} \varphi^{j} \in D^{\times}$with $l \in \mathbb{Z}$ and $d_{j} \in \mu_{q^{n}-1}\left(K_{n}\right) \cup\{0\}$. Then, let $d$ act on $B_{n}$ by

$$
d^{*}: B_{n} \rightarrow B_{n} ; \boldsymbol{X}_{i} \mapsto \widehat{\mathcal{G}_{0}} \sum_{j=l}^{\infty} d_{j} \boldsymbol{X}_{i}^{q^{j}} \quad \text { for } 1 \leq i \leq n
$$

Now, we give a right action of $G^{0}$ on $\mathbf{M}_{\infty, \mathcal{O}_{\mathbf{C}}}^{(0)}$ using (1.6) and (1.7). Let $(g, d, 1) \in G^{0}$. We set

$$
\gamma(g, d)=\operatorname{det}(g) \operatorname{Nrd}_{D / K}(d)^{-1} \in \mathcal{O}_{K}^{\times}
$$

We put $t=\left(t^{q^{-m}}\right)_{m \geq 0}$. Let $(g, d, 1)$ act on $\mathbf{M}_{\infty, \mathcal{O}_{\mathbf{C}}}^{(0)}$ by

$$
A^{\alpha} \rightarrow A^{\gamma(g, d)^{-1} \alpha} ; \boldsymbol{X}_{i} \mapsto(g, d) \cdot \boldsymbol{X}_{i} \text { for } 1 \leq i \leq n,
$$

where $\alpha \in \mathcal{O}_{K}^{\times}$. This is well-defined, because the equation

$$
\delta\left((g, d) \cdot \boldsymbol{X}_{1}, \ldots,(g, d) \cdot \boldsymbol{X}_{n}\right)=\operatorname{Art}_{K}(\alpha)(\boldsymbol{t})
$$

is equivalent to $\delta\left(\boldsymbol{X}_{1}, \ldots, \boldsymbol{X}_{n}\right)=\operatorname{Art}_{K}\left(\gamma(g, d)^{-1} \alpha\right)(\boldsymbol{t})$. Let $\left(1, \varphi^{-n_{\sigma}}, \sigma\right) \in G^{0}$ act on $\mathbf{M}_{\infty, \mathcal{O}_{\mathbf{C}}}^{(0)}$ by

$$
A^{\alpha} \rightarrow A^{a_{K}(\sigma) \alpha} ; \boldsymbol{X}_{i} \mapsto \boldsymbol{X}_{i}, \quad x \mapsto \sigma(x) \quad \text { for } 1 \leq i \leq n \text { and } x \in \mathcal{O}_{\mathbf{C}},
$$

where $\alpha \in \mathcal{O}_{K}^{\times}$. Thus, we have a right action of $G^{0}$ on $\mathbf{M}_{\infty, \mathcal{O}_{\mathbf{C}}}$, which induces a right action on $\mathcal{M}_{\infty, \bar{\eta}}^{(0)}(\mathbf{C})=\mathcal{M}_{\infty}(\mathbf{C})$.

Remark 1.4 For $a \in K^{\times}$, the action of $(a, a, 1) \in G^{0}$ on $\mathbf{M}_{\infty, \mathcal{O}_{\mathbf{C}}}$ is trivial by the definition.

\subsection{CM points}

We recall the notion of CM points from [5, 3.1]. Let $L$ be a finite extension of $K$ of degree $n$ inside $\mathbf{C}$.

Definition 1.5 A deformation $\mathcal{G}$ of $\mathcal{G}_{0}$ over $\mathcal{O}_{\mathbf{C}}$ has $\mathrm{CM}$ by $L$ if there is an isomorphism $L \stackrel{\sim}{\rightarrow} \operatorname{End}(\mathcal{G}) \otimes \mathcal{O}_{K} K$ as $K$-algebras such that the induced map $L \rightarrow \operatorname{End}($ Lie $\mathcal{G}) \otimes \mathcal{O}_{K}$ $K \simeq \mathbf{C}$ coincides with the natural embedding $L \subset \mathbf{C}$. 
We say that a point of $\mathcal{M}_{\infty}(\mathbf{C})$ has $\mathrm{CM}$ by $L$ if the corresponding deformation over $\mathcal{O}_{\mathrm{C}}$ has $\mathrm{CM}$ by $L$.

Let $\xi \in \mathcal{M}_{\infty}(\mathbf{C})$ be a point that has CM by $L$. Let $(\mathcal{G}, \phi, \iota)$ be the triple corresponding to $\xi$. Then we have embeddings $i_{M, \xi}: L \rightarrow M_{n}(K)$ and $i_{D, \xi}: L \rightarrow D$ characterized by the commutative diagrams

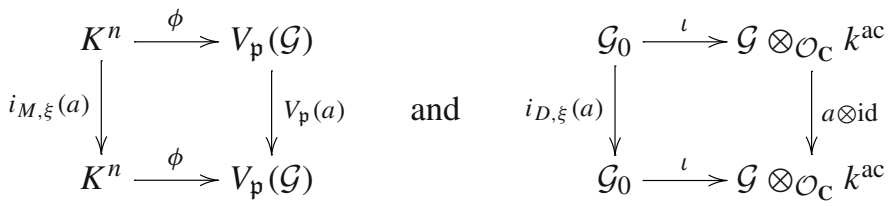

in the isogeny category for $a \in L$. We put $i_{\xi}=\left(i_{M, \xi}, i_{D, \xi}\right): L \rightarrow M_{n}(K) \times D$. We put

$$
\left(G L_{n}(K) \times D^{\times}\right)^{0}=\left\{(g, d) \in G L_{n}(K) \times D^{\times} \mid(g, d, 1) \in G^{0}\right\} .
$$

Lemma $1.6\left(\left[5\right.\right.$, Lemma 3.1.2]) The group $\left(G L_{n}(K) \times D^{\times}\right)^{0}$ acts transitively on the set of the points of $\mathcal{M}_{\infty}(\mathbf{C})$ that have $C M$ by L. For $\xi \in \mathcal{M}_{\infty}(\mathbf{C})$ that has $C M$ by $L$, the stabilizer of $\xi$ in $\left(G L_{n}(K) \times D^{\times}\right)^{0}$ is $i_{\xi}\left(L^{\times}\right)$.

\section{Good reduction of affinoids}

\subsection{Construction of affinoids}

We take a uniformizer $\varpi$ of $K$. Let $r \in \mu_{q-1}(K)$. We put $\varpi_{r}=r \varpi$. We take $\varphi_{r} \in \mathbf{C}$ such that $\varphi_{r}^{n}=\varpi_{r}$. We apply results in Sect. 1 replacing $\varpi$ with $\varpi_{r}$. We put $L_{r}=K\left(\varphi_{r}\right)$. By the $\mathcal{O}_{K}$-algebra embedding $\mathcal{O}_{L_{r}} \rightarrow \mathcal{O}_{D}$ defined by $\varphi_{r} \mapsto \varphi$, we view $\mathcal{G}_{0}$ as a formal $\mathcal{O}_{L_{r}}$-module of height 1 . Let $\mathcal{G}_{r}$ be a lift of $\mathcal{G}_{0}$ to $\mathcal{O}_{\widehat{L}_{r}^{\text {ur }}}$ as a formal $\mathcal{O}_{L_{r}}$-module. We take a compatible system $\left\{t_{r, m}\right\}_{m \geq 1}$ in $\mathbf{C}$ such that

$$
t_{r, 1} \neq 0, \quad\left[\varphi_{r}\right]_{\mathcal{G}_{r}}\left(t_{r, 1}\right)=0, \quad\left[\varphi_{r}\right]_{\mathcal{G}_{r}}\left(t_{r, m}\right)=t_{r, m-1}
$$

for $m \geq 2$. We put

$$
\varphi_{M, r}=\left(\begin{array}{cc}
\mathbf{0} & I_{n-1} \\
\varpi_{r} & \mathbf{0}
\end{array}\right) \in M_{n}(K)
$$

and $\varphi_{D, r}=\varphi \in D$. For $\xi \in \mathcal{M}_{\infty, \bar{\eta}}^{(0)}(\mathbf{C})$, we write $\left(\boldsymbol{\xi}_{1}, \ldots, \boldsymbol{\xi}_{n}\right)$ for the coordinate of $\xi$ with respect to $\left(\boldsymbol{X}_{1}, \ldots, \boldsymbol{X}_{n}\right)$, where $\boldsymbol{\xi}_{i}=\left(\xi_{i}^{q^{-j}}\right)_{j \geq 0}$ for $1 \leq i \leq n$.

Lemma 2.1 There exists $\xi_{r} \in \mathcal{M}_{\infty, \bar{\eta}}^{(0)}(\mathbf{C})$ such that

$$
\xi_{r, i}^{q^{-j}}=\lim _{m \rightarrow \infty} t_{r, m}^{q^{m-i-j}} \in \mathcal{O}_{\mathbf{C}}
$$


for $1 \leq i \leq n$ and $j \geq 0$. Further, we have the following:

(1) $\xi_{r}$ has $C M$ by $L_{r}$.

(2) We have $i_{\xi_{r}}\left(\varphi_{r}\right)=\left(\varphi_{M, r}, \varphi_{D, r}\right) \in M_{n}(K) \times D$.

(3) $\boldsymbol{\xi}_{r, i}=\xi_{r, i+1}^{q}$ for $1 \leq i \leq n-1$.

(4) $v\left(\xi_{r, i}\right)=1 /\left(n q^{i-1}(q-1)\right)$ for $1 \leq i \leq n$.

Proof This is proved in the same way as [12, Lemma 2.2].

We take $\xi_{r}$ as in Lemma 2.1. We can replace the choice of (1.2) so that $\delta\left(\xi_{1}, \ldots, \xi_{n}\right)=\boldsymbol{t}$. Then we have $\xi_{r} \in \mathcal{M}_{\infty, \bar{\eta}, 1}^{(0)}$. Let $\mathcal{D}_{\mathbf{C}}^{n \text {,perf }}$ be the generic fiber of Spf $\mathcal{O}_{\mathbf{C}}\left[\left[X_{1}^{1 / q^{\infty}}, \ldots, X_{n}^{1 / q^{\infty}}\right]\right]$. We consider $\mathcal{M}_{\infty, \bar{\eta}, 1}^{(0)}$ as a subspace of $\mathcal{D}_{\mathbf{C}}^{n, \text { perf }}$ by (1.4). We put $\boldsymbol{\eta}_{r}=\boldsymbol{\xi}_{r, 1}^{q-1}$ and write $\boldsymbol{\eta}_{r}=\left(\eta_{r}^{q^{-j}}\right)_{j \geq 0}$. Note that $v\left(\boldsymbol{\eta}_{r}\right)=1 / n$. We write $n=p^{e} n^{\prime}$ with $\operatorname{gcd}\left(p, n^{\prime}\right)=1$. We assume that $e \geq 1$ in the sequel, since the case where $e=0$ is already studied in [12]. We put

$$
\varepsilon_{0}= \begin{cases}\left(n^{\prime}+1\right) / 2 & \text { if } p^{e}=2 \\ 0 & \text { if } p^{e} \neq 2\end{cases}
$$

We take $q$-th power compatible systems $\boldsymbol{\theta}_{r}=\left(\theta_{r}^{q^{-j}}\right)_{j \geq 0}$ and $\lambda_{r}=\left(\lambda_{r}^{q^{-j}}\right)_{j \geq 0}$ in $\mathbf{C}$ satisfying

$$
\boldsymbol{\theta}_{r}^{p^{2 e}}+\boldsymbol{\eta}_{r}^{p^{e}-1}\left(\boldsymbol{\theta}_{r}+1\right)=0, \quad \lambda_{r}^{q}-\eta_{r}^{q-1}\left(\lambda_{r}-\boldsymbol{\theta}_{r}^{p^{e}}\left(\boldsymbol{\theta}_{r}+1\right)+\varepsilon_{0} \boldsymbol{\eta}_{r}\right)=0 .
$$

Note that

$$
v\left(\boldsymbol{\theta}_{r}\right)=\frac{p^{e}-1}{n p^{2 e}}, \quad v\left(\boldsymbol{\lambda}_{r}\right)=\frac{1}{n}\left(1-\frac{1}{q p^{e}}\right) .
$$

We define $\xi_{r}^{\prime} \in \mathcal{D}_{\mathbf{C}}^{n, \text { perf }}$ by

$$
\begin{aligned}
& \boldsymbol{\xi}_{r, 1}^{\prime}=\boldsymbol{\xi}_{r, 1}\left(1+\boldsymbol{\theta}_{r}\right), \quad \boldsymbol{\xi}_{r, i+1}^{\prime}=\boldsymbol{\xi}_{r, i}^{\frac{1}{q}} \text { for } 1 \leq i \leq n-2, \\
& \boldsymbol{\xi}_{r, n}^{\prime}=\boldsymbol{\xi}_{r, n-1}^{\prime \frac{1}{q}}\left(\left(1+\boldsymbol{\theta}_{r}\right)^{-n}\left(1+n^{\prime} \lambda_{r}\right)\right)^{\frac{1}{q^{n-1}}}
\end{aligned}
$$

Proposition 2.2 There uniquely exists $\xi_{r}^{0} \in \mathcal{M}_{\infty, \bar{\eta}, 1}^{(0)}$ satisfying

$$
\xi_{r, i}^{0}=\xi_{r, i}^{\prime} \quad \text { for } 1 \leq i \leq n-1, \quad \xi_{r, n}^{0} \equiv \xi_{r, n}^{\prime} \quad \bmod >\frac{q^{2}-q+1}{n q^{n-1}(q-1)}
$$

Proof We have

$$
\delta\left(\xi_{r}^{\prime}\right) \equiv t \quad \bmod >\frac{1}{q-1}+\frac{1}{n} .
$$

Hence, we see the claim by Newton's method. 
Remark 2.3 The key ingredients for the construction of $\xi_{r}^{0}$ are the elements $\boldsymbol{\theta}_{r}$ and $\lambda_{r}$ defined by (2.1). Up to some difference of normalizations, these elements are analogues of $\beta_{\zeta}$ and $\gamma_{\zeta}$ in $[13, \S 2.2]$, which are generators of a field extension used in a construction of a Langlands parameter there.

We take $\xi_{r}^{0}$ as in Proposition 2.2. We put $\boldsymbol{x}_{i}=\boldsymbol{X}_{i} / \boldsymbol{\xi}_{r, i}^{0}$ for $1 \leq i \leq n$. We define $\mathcal{X}_{r} \subset \mathcal{M}_{\infty, \bar{\eta}, 1}^{(0)}$ by

$$
\begin{aligned}
& v\left(\frac{\boldsymbol{x}_{i}}{\boldsymbol{x}_{i+1}}-\left(\frac{\boldsymbol{x}_{n-1}}{\boldsymbol{x}_{n}}\right)^{q^{n-1-i}}\right) \geq \frac{1}{2 n q^{i}} \text { for } 1 \leq i \leq n-2, \\
& v\left(\boldsymbol{x}_{i}-1\right) \geq \frac{1}{n q^{n-1}\left(p^{e}+1\right)} \text { for } n-1 \leq i \leq n .
\end{aligned}
$$

The definition of $\mathcal{X}_{r}$ is independent of the choice of $\boldsymbol{\theta}_{r}$ and $\lambda_{r}$. We define $\mathcal{B}_{r} \subset \mathcal{D}_{\mathbf{C}}^{n \text {,perf }}$ by the same condition (2.2).

\subsection{Formal models of affinoids}

Let $\left(\boldsymbol{X}_{1}, \ldots, \boldsymbol{X}_{n}\right)$ be the coordinate of $\mathcal{B}_{r}$. We put $h\left(\boldsymbol{X}_{1}, \ldots, \boldsymbol{X}_{n}\right)=\prod_{i=1}^{n} \boldsymbol{X}_{i}^{q^{i-1}}$. Further, we put

$$
\begin{aligned}
f\left(\boldsymbol{X}_{1}, \ldots, \boldsymbol{X}_{n}\right) & =1-\frac{\delta\left(\boldsymbol{X}_{1}, \ldots, \boldsymbol{X}_{n}\right)}{h\left(\boldsymbol{X}_{1}, \ldots, \boldsymbol{X}_{n}\right)} \\
f_{0}\left(\boldsymbol{X}_{1}, \ldots, \boldsymbol{X}_{n}\right) & =\sum_{i=1}^{n-1}\left(\frac{\boldsymbol{X}_{i}}{\boldsymbol{X}_{i+1}}\right)^{q^{i-1}(q-1)}+\left(\frac{\boldsymbol{X}_{n}^{q^{n}}}{\boldsymbol{X}_{1}}\right)^{\frac{q-1}{q}} .
\end{aligned}
$$

We simply write $f(\boldsymbol{X})$ for $f\left(\boldsymbol{X}_{1}, \ldots, \boldsymbol{X}_{n}\right)$, and $f\left(\boldsymbol{\xi}_{r}\right)$ for $f\left(\boldsymbol{\xi}_{r, 1}, \ldots, \boldsymbol{\xi}_{r, n}\right)$. We will use the similar notations also for other functions. We put

$$
\boldsymbol{S}=f_{0}(\boldsymbol{X})-f_{0}\left(\boldsymbol{\xi}_{r}^{0}\right)
$$

Lemma 2.4 We have

$$
f(\boldsymbol{X}) \equiv f_{0}(\boldsymbol{X}) \quad \bmod _{>} \frac{q-1}{n q} \text { and } \boldsymbol{S} \equiv f(\boldsymbol{X})-f\left(\xi_{r}^{0}\right) \quad \bmod _{>} \frac{1}{n}
$$

Proof We put

$$
\begin{aligned}
f_{1}\left(\boldsymbol{X}_{1}, \ldots, \boldsymbol{X}_{n}\right)= & \sum_{i=1}^{n-3} \sum_{j=i+2}^{n-1}\left(\frac{\boldsymbol{X}_{i}}{\boldsymbol{X}_{i+1}}\right)^{q^{i-1}(q-1)}\left(\frac{\boldsymbol{X}_{j}}{\boldsymbol{X}_{j+1}}\right)^{q^{j-1}(q-1)} \\
& +\left(\frac{\boldsymbol{X}_{n}^{q^{n}}}{\boldsymbol{X}_{1}}\right)^{\frac{q-1}{q}} \sum_{i=1}^{n-3}\left(\frac{\boldsymbol{X}_{i+1}}{\boldsymbol{X}_{i+2}}\right)^{q^{i}(q-1)}
\end{aligned}
$$


We note that $\left(\boldsymbol{X}_{1}, \ldots, \boldsymbol{X}_{n}\right)$ satisfies the assumption of Lemma 1.3 by the definition of $\mathcal{B}_{r}$ and Lemma 2.1 (4). Then we see that

$$
f(\boldsymbol{X}) \equiv 1-\frac{\delta_{0}^{\prime}(\boldsymbol{X})}{h(\boldsymbol{X})} \equiv f_{0}(\boldsymbol{X})-f_{1}(\boldsymbol{X}) \quad \bmod _{>} \frac{1}{n}
$$

using Lemma 1.3 and the definition of $\delta_{0}^{\prime}$. The claims follow from this, because

$$
v\left(f_{1}(\boldsymbol{X})\right) \geq \frac{2(q-1)}{n q} \text { and } v\left(f_{1}(\boldsymbol{X})-f_{1}\left(\xi_{r}^{0}\right)\right)>\frac{2(q-1)}{n q}
$$

hold.

We put $\boldsymbol{s}_{i}=\left(\boldsymbol{x}_{i} / \boldsymbol{x}_{i+1}\right)^{q^{i}(q-1)}$ for $1 \leq i \leq n-1$, and

$$
\boldsymbol{s}_{i} \boldsymbol{s}_{n-1}^{-1}=1+\boldsymbol{Y}_{i} \text { for } 1 \leq i \leq n-2, \quad \boldsymbol{s}_{n-1}=1+\boldsymbol{Y}_{n-1}
$$

We put $m=\operatorname{gcd}(e, f)$ and

$$
\boldsymbol{z}=\sum_{i=0}^{\frac{e}{m}-1}\left(\frac{\boldsymbol{\theta}_{r}^{p^{e}} \boldsymbol{Y}_{n-1}}{\boldsymbol{\eta}_{r}}\right)^{p^{i m}}-\frac{1}{n^{\prime}} \sum_{i=0}^{\frac{f}{m}-1}\left(\frac{\boldsymbol{S}}{\boldsymbol{\eta}_{r}}\right)^{p^{i m}}
$$

We put $f=m_{0}$ and $e=m_{1}$. We define $m_{2}, \ldots, m_{N+1}$ by the Euclidean algorithm as follows: We have

$$
\begin{aligned}
& m_{i-1}=n_{i} m_{i}+m_{i+1} \quad \text { with } n_{i} \geq 0 \text { and } 0 \leq m_{i+1}<m_{i} \text { for } 1 \leq i \leq N, \\
& m_{N}=m, \quad m_{N+1}=0 .
\end{aligned}
$$

We put

$$
\boldsymbol{T}_{0}=\frac{\boldsymbol{\theta}_{r}^{p^{e}} \boldsymbol{Y}_{n-1}}{\boldsymbol{\eta}_{r}}, \quad \boldsymbol{T}_{1}=\frac{-\boldsymbol{S}}{n^{\prime} \boldsymbol{\eta}_{r}}
$$

and define $\boldsymbol{T}_{2}, \ldots, \boldsymbol{T}_{N}$ by

$$
\boldsymbol{T}_{i+1}=\boldsymbol{T}_{i-1}+\sum_{j=0}^{n_{i}-1} \boldsymbol{T}_{i}^{p^{j m_{i}+m_{i+1}}} \quad \text { for } 1 \leq i \leq N-1
$$

Then we see that

$$
\boldsymbol{z}=\sum_{j=0}^{\frac{m_{i+1}}{m}-1} \boldsymbol{T}_{i}^{p^{j m}}+\sum_{j=0}^{\frac{m_{i}}{m}-1} \boldsymbol{T}_{i+1}^{p^{j m}} \quad \text { for } 1 \leq i \leq N-1
$$


inductively by (2.7). We see also that

$$
(-1)^{N-i} \boldsymbol{T}_{i}=\sum_{j=0}^{\frac{m_{i}}{m}-1} \boldsymbol{T}_{N}^{p^{j m}}+P_{i}(\boldsymbol{z})
$$

with some $P_{i}(x) \in \mathbb{Z}[x]$ for $0 \leq i \leq N-1$. We put

$$
\boldsymbol{Y}=\frac{(-1)^{N} \boldsymbol{\eta}_{r}}{\boldsymbol{\theta}_{r}^{p^{e}}} \boldsymbol{T}_{N}^{p^{f-m}}
$$

Then we have

$$
\boldsymbol{Y} \equiv \boldsymbol{Y}_{n-1} \quad \bmod >\frac{1}{n\left(p^{e}+1\right)}
$$

by (2.9) and (2.10). We define a subaffinoid $\mathcal{B}_{r}^{\prime} \subset \mathcal{B}_{r}$ by $v(z) \geq 0$. We choose a square $\operatorname{root} \eta_{r}^{1 / 2}=\left(\eta_{r}^{q^{-j} / 2}\right)_{j \geq 0}$ and a $\left(p^{e}+1\right)$-st root $\eta_{r}^{1 /\left(p^{e}+1\right)}=\left(\eta_{r}^{q^{-j} /\left(p^{e}+1\right)}\right)_{j \geq 0}$ of $\eta_{r}$ compatibly. We set

$$
\begin{aligned}
& \boldsymbol{Y}_{i}=\boldsymbol{\eta}_{r}^{1 / 2} \boldsymbol{y}_{i} \text { with } \boldsymbol{y}_{i}=\left(y_{i}^{q^{-j}}\right)_{j \geq 0} \text { for } 1 \leq i \leq n-2, \\
& \boldsymbol{Y}=\boldsymbol{\eta}_{r}^{1 /\left(p^{e}+1\right)} \boldsymbol{y} \text { with } \boldsymbol{y}=\left(y^{q^{-j}}\right)_{j \geq 0}
\end{aligned}
$$

on $\mathcal{B}_{r}^{\prime}$. Let $\mathcal{B}$ be the generic fiber of $\operatorname{Spf} \mathcal{O}_{\mathbf{C}}\left\langle y^{1 / q^{\infty}}, y_{1}^{1 / q^{\infty}}, \ldots, y_{n-2}^{1 / q^{\infty}}, z^{1 / q^{\infty}}\right\rangle$. The parameters $\boldsymbol{y}, \boldsymbol{y}_{1}, \ldots, \boldsymbol{y}_{n-2}, \boldsymbol{z}$ give the morphism $\Theta: \mathcal{B}_{r}^{\prime} \rightarrow \mathcal{B}$. We simply say an analytic function on $\mathcal{B}$ for a $q$-th power compatible system of analytic functions on $\mathcal{B}$. We put

$$
1+\boldsymbol{\theta}_{r}^{\prime}=\left(1+\boldsymbol{\theta}_{r}\right)^{-n}\left(1+n^{\prime} \lambda_{r}\right)\left(\frac{\boldsymbol{\xi}_{n}^{0}}{\boldsymbol{\xi}_{n}^{\prime}}\right)^{q^{n-1}} .
$$

Lemma 2.5 The morphism $\Theta$ is an isomorphism.

Proof We will construct the inverse morphism of $\Theta$. We can write $\boldsymbol{Y}_{n-1}$ and $\boldsymbol{S}$ as analytic functions on $\mathcal{B}$ by (2.8), (2.9), (2.10) and (2.12). Then we can write $\boldsymbol{x}_{i} / \boldsymbol{x}_{i+1}$ as an analytic function on $\mathcal{B}$ by (2.6). By (2.4) and (2.5), we have

$$
\begin{aligned}
\frac{\eta_{r}^{-(q-1)} S^{q}}{\left(1+\boldsymbol{\theta}_{r}\right)^{(q-1)^{2}}}= & \sum_{i=1}^{n-2}\left(\boldsymbol{s}_{i}-1\right)+\frac{\boldsymbol{s}_{n-1}-1}{\left(1+\boldsymbol{\theta}_{r}^{\prime}\right)^{q-1}} \\
& +\left(1+\boldsymbol{\theta}_{r}^{\prime}\right)^{q(q-1)}\left(\boldsymbol{x}_{n}^{(q-1)\left(q^{n}-1\right)} \prod_{i=1}^{n-1}\left(\boldsymbol{x}_{i}^{-1} \boldsymbol{x}_{i+1}\right)-1\right) .
\end{aligned}
$$

By this equation, we can write $\boldsymbol{x}_{n}$ as an analytic functions on $\mathcal{B}$. Hence, we have the inverse morphism of $\Theta$. 
We put

$$
\delta_{\mathcal{B}}\left(y, y_{1}, \ldots, y_{n-2}, z\right)=\left(\left.\delta\right|_{\mathcal{B}_{r}^{\prime}}\right) \circ \Theta^{-1}
$$

equipped with its $q^{j}$-th $\operatorname{root} \delta_{\mathcal{B}}^{q^{-j}}$ for $j \geq 0$. We put

$$
\mathfrak{X}_{r}=\operatorname{Spf} \mathcal{O}_{\mathbf{C}}\left\langle y^{1 / q^{\infty}}, y_{1}^{1 / q^{\infty}}, \ldots, y_{n-2}^{1 / q^{\infty}}, z^{1 / q^{\infty}}\right\rangle /\left(\delta_{\mathcal{B}}^{q^{-j}}-t^{q^{-j}}\right)_{j \geq 0}^{-} .
$$

Let $\overline{\mathfrak{X}}_{r}$ denote the special fiber of $\mathfrak{X}_{r}$.

Theorem 2.6 The formal scheme $\mathfrak{X}_{r}$ is a formal model of $\mathcal{X}_{r}$, and $\overline{\mathfrak{X}}_{r}$ is isomorphic to the perfection of the affine smooth variety defined by

$$
z^{p^{m}}-z=y^{p^{e}+1}-\frac{1}{n^{\prime}} \sum_{1 \leq i \leq j \leq n-2} y_{i} y_{j} \quad \text { in } \mathbb{A}_{k^{\mathrm{ac}}}^{n}
$$

Proof Let $\left(\boldsymbol{X}_{1}, \ldots, \boldsymbol{X}_{n}\right)$ be the coordinate of $\mathcal{B}_{r}$. By Lemma 2.4, we have

$$
v(f(\boldsymbol{X})) \geq \frac{q-1}{n q} \text { and } v(S)>\frac{q-1}{n q} .
$$

We have

$$
h(\boldsymbol{X})^{q-1}=\left(\frac{\boldsymbol{X}_{n}^{q^{n}}}{\boldsymbol{X}_{1}}\right) \prod_{i=1}^{n-1}\left(\frac{\boldsymbol{X}_{i}}{\boldsymbol{X}_{i+1}}\right)^{q^{i}}
$$

We have

$$
\left(\frac{\boldsymbol{X}_{n}^{q^{n}}}{\boldsymbol{X}_{1}}\right)^{q-1}=\left(\boldsymbol{\eta}_{r}\left(1+\boldsymbol{\theta}_{r}\right)^{q-1}\left(1+\boldsymbol{\theta}_{r}^{\prime}\right)^{q}\right)^{q-1}\left(\frac{h(\boldsymbol{X})}{h\left(\boldsymbol{\xi}_{r}^{0}\right)}\right)^{(q-1)^{2}} \prod_{i=1}^{n-1} \boldsymbol{s}_{i}^{-1}
$$

by (2.15). We put

$$
R(\boldsymbol{X})=\frac{1-f\left(\xi_{r}^{0}\right)}{1-f(\boldsymbol{X})}-(1+\boldsymbol{S}) .
$$

Then we have $v(R(\boldsymbol{X}))>1 / n$ by Lemma 2.4 and (2.14). The equation $\delta(\boldsymbol{X})=\delta\left(\boldsymbol{\xi}_{r}^{0}\right)$ is equivalent to

$$
\left(\frac{\boldsymbol{X}_{n}^{q^{n}}}{\boldsymbol{X}_{1}}\right)^{q-1}=\left(\eta_{r}\left(1+\boldsymbol{\theta}_{r}\right)^{q-1}\left(1+\boldsymbol{\theta}_{r}^{\prime}\right)^{q}\right)^{q-1}(1+\boldsymbol{S}+R(\boldsymbol{X}))^{(q-1)^{2}} \prod_{i=1}^{n-1} \boldsymbol{s}_{i}^{-1}
$$

by (2.3), (2.16) and (2.17). We put

$$
F(\boldsymbol{X})=\left(1+\boldsymbol{\theta}_{r}^{\prime}\right)^{q(q-1)}(1+\boldsymbol{S}+R(\boldsymbol{X}))^{(q-1)^{2}} \prod_{i=1}^{n-1} \boldsymbol{s}_{i}^{-1}
$$


The equation (2.18) is equivalent to

$$
f_{0}(\boldsymbol{X})^{q}=\eta_{r}^{q-1}\left(1+\boldsymbol{\theta}_{r}\right)^{(q-1)^{2}}\left(\sum_{i=1}^{n-2} \boldsymbol{s}_{i}+\frac{\boldsymbol{s}_{n-1}}{\left(1+\boldsymbol{\theta}_{r}^{\prime}\right)^{q-1}}+F(\boldsymbol{X})\right) .
$$

The equation (2.19) is equivalent to

$$
\boldsymbol{S}^{q}=\eta_{r}^{q-1}\left(1+\boldsymbol{\theta}_{r}\right)^{(q-1)^{2}}\left(\sum_{i=1}^{n-2}\left(\boldsymbol{s}_{i}-1\right)+\frac{\boldsymbol{s}_{n-1}-1}{\left(1+\boldsymbol{\theta}_{r}^{\prime}\right)^{q-1}}+F(\boldsymbol{X})-F\left(\boldsymbol{\xi}_{r}^{0}\right)\right) .
$$

We put

$$
\begin{aligned}
R_{1}(\boldsymbol{X})= & \left(1+\boldsymbol{\theta}_{r}\right)^{(q-1)^{2}}\left(\sum_{i=1}^{n-2}\left(\boldsymbol{s}_{i}-1\right)+\frac{\boldsymbol{s}_{n-1}-1}{\left(1+\boldsymbol{\theta}_{r}^{\prime}\right)^{q-1}}+F(\boldsymbol{X})-F\left(\boldsymbol{\xi}_{r}^{0}\right)\right) \\
& -\left(\boldsymbol{S}+\sum_{1 \leq i \leq j \leq n-2} \boldsymbol{Y}_{i} \boldsymbol{Y}_{j}-n^{\prime}\left(\boldsymbol{Y}_{n-1}^{p^{e}+1}+\left(1+\boldsymbol{\theta}_{r}\right) \boldsymbol{Y}_{n-1}^{p^{e}}+\boldsymbol{\theta}_{r}^{p^{e}} \boldsymbol{Y}_{n-1}\right)\right)
\end{aligned}
$$

Then we have $v\left(R_{1}(\boldsymbol{X})\right)>1 / n$. The equation (2.20) is equivalent to

$$
\boldsymbol{S}^{q}=\eta_{r}^{q-1}\left(\boldsymbol{S}+\sum_{1 \leq i \leq j \leq n-2} \boldsymbol{Y}_{i} \boldsymbol{Y}_{j}-n^{\prime}\left(\boldsymbol{Y}_{n-1}^{p^{e}+1}+\left(1+\boldsymbol{\theta}_{r}\right) \boldsymbol{Y}_{n-1}^{p^{e}}+\boldsymbol{\theta}_{r}^{p^{e}} \boldsymbol{Y}_{n-1}\right)+R_{1}(\boldsymbol{X})\right)
$$

The equation (2.21) is equivalent to

$$
z^{p^{m}}-z=\eta_{r}^{-1}\left(\boldsymbol{Y}_{n-1}^{p^{e}+1}-\frac{1}{n^{\prime}} \sum_{1 \leq i \leq j \leq n-2} \boldsymbol{Y}_{i} \boldsymbol{Y}_{j}-\frac{R_{1}(\boldsymbol{X})}{n^{\prime}}\right)
$$

As a result, $\delta(\boldsymbol{X})=\delta\left(\boldsymbol{\xi}_{L}\right)$ is equivalent to (2.22) on $\mathcal{B}_{r}$. By Lemma 2.4 and (2.22), we have $v(z) \geq 0$ on $\mathcal{X}_{r}$. This implies $\mathcal{X}_{r} \subset \mathcal{B}_{r}^{\prime}$. We have the first claim by Lemma 2.5 and the construction of $\mathfrak{X}_{r}$. The second claim follows from (2.11) and (2.22).

Remark 2.7 If $n=p=2$, then the smooth compactification of the curve over $k$ defined by (2.13) is the supersingular elliptic curve, which appears as an irreducible component of a semi-stable reduction of a one-dimensional Lubin-Tate space in [15] and [11].

\section{Group action on the reductions}

Action of $G L_{n}$ and $D^{\times}$Let $\mathfrak{I} \subset M_{n}\left(\mathcal{O}_{K}\right)$ be the inverse image under the reduction map $M_{n}\left(\mathcal{O}_{K}\right) \rightarrow M_{n}(k)$ of the ring consisting of upper triangular matrices in $M_{n}(k)$. 
Lemma 3.1 Let $(g, d, 1) \in G^{0}$. We take the integer $l$ such that $d \varphi_{D, r}^{-l} \in \mathcal{O}_{D}^{\times}$. Let $\left(\boldsymbol{X}_{1}, \ldots, \boldsymbol{X}_{n}\right)$ be the coordinate of $\mathcal{X}_{r}$. Assume $v\left((g, d) \cdot \boldsymbol{X}_{i}\right)=v\left(\boldsymbol{X}_{i}\right)$ for $1 \leq i \leq n$ at some point of $\mathcal{X}_{r}$. Then we have $(g, d) \in\left(\varphi_{M, r}, \varphi_{D, r}\right)^{l}\left(\mathfrak{I}^{\times} \times \mathcal{O}_{D}^{\times}\right)$.

Proof This is proved in the same way as [12, Lemma 3.1].

We put

$$
\mathbf{g}_{r}=\left(\varphi_{M, r}, \varphi_{D, r}, 1\right) \in G \text {. }
$$

We put

$$
\varepsilon_{1}= \begin{cases}1 & \text { if } p^{e}=2 \\ 0 & \text { if } p^{e} \neq 2\end{cases}
$$

For $a \in k^{\mathrm{ac}}$, we simply write $a$ also for the $q$-th power compatible system $\left(a^{q^{-j}}\right)_{j \geq 0}$.

Proposition 3.2 (1) The action of $\mathbf{g}_{r}$ stabilizes $\mathcal{X}_{r}$, and induces the automorphism of $\overline{\mathfrak{X}}_{r}$ defined by

$$
\begin{aligned}
& \left(\boldsymbol{z}, \boldsymbol{y},\left(\boldsymbol{y}_{i}\right)_{1 \leq i \leq n-2}\right) \\
& \mapsto\left(z+\varepsilon_{1}\left(\boldsymbol{y}_{n-2}+1\right), \boldsymbol{y},-\sum_{i=1}^{n-3} \boldsymbol{y}_{i}-2 \boldsymbol{y}_{n-2}+\varepsilon_{1},\left(\boldsymbol{y}_{i-1}-\boldsymbol{y}_{n-2}+\varepsilon_{1}\right)_{2 \leq i \leq n-2}\right) .
\end{aligned}
$$

(2) Assume $p^{e} \neq 2$. Let $g_{r} \in G L_{n-1}(k)$ be the matrix corresponding to the action of $\mathbf{g}_{r}$ on $\left(\boldsymbol{y},\left(\boldsymbol{y}_{i}\right)_{1 \leq i \leq n-2}\right)$ in (3.3). Then, $\operatorname{det}\left(g_{r}\right)=(-1)^{n-1}$.

Proof By (1.6) and (1.7), we have

$$
\mathbf{g}_{r}^{*} \boldsymbol{X}_{1}=\boldsymbol{X}_{n}^{q^{n-1}}, \quad \mathbf{g}_{r}^{*} \boldsymbol{X}_{i}=\boldsymbol{X}_{i-1}^{\frac{1}{q}} \quad \text { for } 2 \leq i \leq n
$$

By (3.4), we have $\mathbf{g}_{r}^{*}(h(\boldsymbol{X}))=h(\boldsymbol{X})$. Hence, we have

$$
\mathbf{g}_{r}^{*} \boldsymbol{S} \equiv \boldsymbol{S} \quad \bmod _{>} \frac{1}{n}
$$

by (2.3), (2.5) and Lemma 2.4. By (2.18) and (3.4), we have

$$
\mathbf{g}_{r}^{*} s_{1} \equiv \prod_{i=1}^{n-1} s_{i}^{-1} \quad \bmod _{>} \frac{1}{2 n}
$$

We have also

$$
\mathbf{g}_{r}^{*} \boldsymbol{s}_{i}=\boldsymbol{s}_{i-1} \text { for } 2 \leq i \leq n-2, \quad \mathbf{g}_{r}^{*} \boldsymbol{s}_{n-1}=\boldsymbol{s}_{n-2}\left(1+\boldsymbol{\theta}_{r}^{\prime}\right)^{1-q}
$$

by (3.4). We have

$$
\mathbf{g}_{r}^{*} \boldsymbol{Y}_{1} \equiv\left(1+\boldsymbol{\theta}_{r}\right)^{n}\left(1+\boldsymbol{Y}_{n-2}\right)^{-2} \prod_{i=1}^{n-3}\left(1+\boldsymbol{Y}_{i}\right)^{-1}-1 \quad \bmod _{>} \frac{1}{2 n}
$$


by (2.6), (3.6) and (3.7). We have also

$$
\begin{aligned}
\mathbf{g}_{r}^{*} \boldsymbol{Y}_{i} & \equiv\left(1+\boldsymbol{\theta}_{r}\right)^{n}\left(1+\boldsymbol{Y}_{i-1}\right)\left(1+\boldsymbol{Y}_{n-2}\right)^{-1}-1 \quad \bmod _{>} \frac{1}{2 n} \quad \text { for } 2 \leq i \leq n-2, \\
\mathbf{g}_{r}^{*} \boldsymbol{Y}_{n-1} & \equiv\left(1+\boldsymbol{\theta}_{r}\right)^{-n}\left(1+\boldsymbol{Y}_{n-2}\right)\left(1+\boldsymbol{Y}_{n-1}\right)-1 \quad \bmod _{>} \frac{1}{p^{e} n}
\end{aligned}
$$

by (3.7). The claim follows from (3.5), (3.8) and (3.9).

Let $\mathfrak{P}$ be the Jacobson radical of the order $\mathfrak{I}$, and $\mathfrak{p}_{D}$ be the maximal ideal of $\mathcal{O}_{D}$. We put

$$
U_{\mathfrak{I}}^{1}=1+\mathfrak{P}, \quad U_{D}^{1}=1+\mathfrak{p}_{D}
$$

and

$$
\left(U_{\mathfrak{I}}^{1} \times U_{D}^{1}\right)^{1}=\left\{(g, d) \in U_{\mathfrak{I}}^{1} \times U_{D}^{1} \mid \operatorname{det}(g)^{-1} \operatorname{Nrd}_{D / K}(d)=1\right\} .
$$

Let $\operatorname{pr}_{\mathcal{O}_{K} / k}: \mathcal{O}_{K} \rightarrow k$ be the reduction map. We put

$$
h_{r}(g, d)=\frac{1}{n^{\prime}}\left(\operatorname{Tr}_{k / \mathbb{F}_{p^{m}}} \circ \operatorname{pr}_{\mathcal{O}_{K} / k}\right)\left(\operatorname{Trd}_{D / K}\left(\varphi_{D, r}^{-1}(d-1)\right)-\operatorname{tr}\left(\varphi_{M, r}^{-1}(g-1)\right)\right)
$$

for $(g, d) \in U_{\mathfrak{I}}^{1} \times U_{D}^{1}$.

Proposition 3.3 The stabilizer of $\mathcal{X}_{r}$ in $G L_{n}(K) \times D^{\times}$is $i_{\xi_{r}}\left(L_{r}^{\times}\right) \cdot\left(U_{\mathfrak{I}}^{1} \times U_{D}^{1}\right)^{1}$. Further, $(g, d) \in\left(U_{\mathfrak{I}}^{1} \times U_{D}^{1}\right)^{1}$ induces the automorphism of $\overline{\mathfrak{X}}_{r}$ defined by

$$
\left(\boldsymbol{z}, \boldsymbol{y},\left(\boldsymbol{y}_{i}\right)_{1 \leq i \leq n-2}\right) \mapsto\left(z+h_{r}(g, d), \boldsymbol{y},\left(\boldsymbol{y}_{i}\right)_{1 \leq i \leq n-2}\right) .
$$

Proof Assume that $(g, d) \in G L_{n}(K) \times D^{\times}$stabilizes $\mathcal{X}_{r}$. Then we have $\operatorname{det}(g)=$ $\operatorname{Nrd}_{D / K}(d)$. We will show that

$$
(g, d) \in i_{\xi_{r}}\left(L_{r}^{\times}\right) \cdot\left(U_{\mathfrak{I}}^{1} \times U_{D}^{1}\right)^{1} .
$$

We have $(g, d) \in\left(\varphi_{M, r}, \varphi_{D, r}\right)^{l}\left(\mathfrak{I}^{\times} \times \mathcal{O}_{D}^{\times}\right)$for some integer $l$ by Lemma 3.1 , since $(g, d)$ stabilizes $\mathcal{X}_{r}$ and we have $v\left(\boldsymbol{X}_{i}\right)=1 /\left(n q^{i-1}(q-1)\right)$ for $1 \leq i \leq n$ at any point of $\mathcal{X}_{r}$ by Lemma 2.1 (4) and (2.2). Further, we may assume that $(g, d) \in \mathfrak{I}^{\times} \times \mathcal{O}_{D}^{\times}$, since we already know that $\left(\varphi_{M, r}, \varphi_{D, r}\right)$ stabilizes $\mathcal{X}_{r}$ by Proposition 3.2 (1).

We write $g=\left(a_{i, j}\right)_{1 \leq i, j \leq n} \in \mathfrak{I}$ and $a_{i, j}=\sum_{l=0}^{\infty} a_{i, j}^{(l)} \varpi_{r}^{l}$ with $a_{i, j}^{(l)} \in \mu_{q-1}(K) \cup$ $\{0\}$. By (1.6), we have

$$
\begin{aligned}
& g^{*} \boldsymbol{X}_{1} \equiv a_{1,1}^{(0)} \boldsymbol{X}_{1}+a_{n, 1}^{(1)} \boldsymbol{X}_{n}^{q^{n}} \quad \bmod _{>} \frac{q}{n(q-1)}, \\
& g^{*} \boldsymbol{X}_{i} \equiv a_{i, i}^{(0)} \boldsymbol{X}_{i}+a_{i-1, i}^{(0)} \boldsymbol{X}_{i-1} \quad \bmod >\frac{1}{n q^{i-2}(q-1)} \quad \text { for } 2 \leq i \leq n .
\end{aligned}
$$


We write $d^{-1}=\sum_{i=0}^{\infty} d_{i} \varphi_{D, r}^{i}$ with $d_{i} \in \mu_{q^{n}-1}\left(K_{n}\right) \cup\{0\}$. We set $\kappa(d)=d_{1} / d_{0}$. By (1.7), we have

$$
d^{*} \boldsymbol{X}_{i} \equiv d_{0} \boldsymbol{X}_{i}\left(1+\kappa(d) \boldsymbol{X}_{i}^{q-1}\right) \quad \bmod _{>} \frac{1}{n q^{i-2}(q-1)} \quad \text { for } 1 \leq i \leq n .
$$

By (2.2), (3.10) and (3.11), we have $(g, d) \in i_{\xi_{r}}\left(\mathcal{O}_{K}^{\times}\right) \cdot\left(U_{\mathfrak{I}}^{1} \times U_{D}^{1}\right)^{1}$. Conversely, any element of $i_{\xi_{r}}\left(L_{r}^{\times}\right) \cdot\left(U_{\mathfrak{I}}^{1} \times U_{D}^{1}\right)^{1}$ stabilizes $\mathcal{X}_{r}$ by Remark 1.4, Proposition 3.2 and the above arguments.

Let $(g, d) \in \mathcal{O}_{K}^{\times} U_{\Im}^{1} \times \mathcal{O}_{D}^{\times}$. We put

$$
\Delta_{g}(\boldsymbol{X})=\sum_{i=1}^{n-1} \frac{a_{i, i+1}^{(0)}}{a_{i+1, i+1}^{(0)}}\left(\frac{\boldsymbol{X}_{i}}{\boldsymbol{X}_{i+1}}\right)^{q^{i}}+\frac{a_{n, 1}^{(1)} \boldsymbol{X}_{n}^{q^{n}}}{a_{1,1}^{(0)} \boldsymbol{X}_{1}}, \quad \Delta_{d}(\boldsymbol{X})=\sum_{i=1}^{n} \kappa(d)^{q^{i-1}} \boldsymbol{X}_{i}^{q^{i-1}(q-1)}
$$

Then, we acquire

$$
f_{0}\left((g, d)^{*} \boldsymbol{X}\right) \equiv f_{0}(\boldsymbol{X})+\Delta_{g}(\boldsymbol{X})+\Delta_{d}(\boldsymbol{X}) \quad \bmod >\frac{1}{n} .
$$

We have

$$
(g, d)^{*} \boldsymbol{S} \equiv \boldsymbol{S}+\Delta_{g}(\boldsymbol{X})+\Delta_{d}(\boldsymbol{X}) \quad \bmod _{>} \frac{1}{n}
$$

by (2.5) and (3.12). We have

$$
(g, d)^{*} s_{i} \equiv s_{i} \quad \bmod \geq \frac{1}{n}
$$

for $1 \leq i \leq n-2$. Let $(g, d) \in\left(U_{\mathfrak{I}}^{1} \times U_{D}^{1}\right)^{1}$. We obtain

$$
(g, d)^{*} z=z+h_{r}(g, d)
$$

by (2.7), (3.13) and (3.14). We can compute the action of $(g, d)$ on $\boldsymbol{y}$ and $\left\{\boldsymbol{y}_{i}\right\}_{1 \leq i \leq n-2}$ by (2.6), (2.11), (2.12) and (3.14).

Action of the Weil group We put $\varphi_{r}^{\prime}=\varphi_{r}^{p^{e}}$ and $E_{r}=K\left(\varphi_{r}^{\prime}\right)$. Let $\sigma \in W_{E_{r}}$ in this paragraph. We put

$$
a_{\sigma}=\operatorname{Art}_{E_{r}}^{-1}(\sigma) \text { and } u_{\sigma}=a_{\sigma} \varphi_{r}^{\prime-n_{\sigma}} \in \mathcal{O}_{E_{r}}^{\times} .
$$

We take

$$
b_{\sigma} \in \mu_{q-1}(K) \text { such that } \bar{b}_{\sigma}^{p^{e}}=\bar{u}_{\sigma} \in k
$$

We put

$$
c_{\sigma}=b_{\sigma}^{-n} \operatorname{Nr}_{E_{r} / K}\left(u_{\sigma}\right) \in U_{K}^{1} .
$$


Let

$$
g_{\sigma}=\left(a_{i, j}\right)_{1 \leq i, j \leq n} \in \mathcal{O}_{K}^{\times} U_{\mathfrak{I}}^{1}
$$

be the element defined by $a_{i, i}=b_{\sigma}$ for $1 \leq i \leq n-1, a_{n, n}=b_{\sigma} c_{\sigma}$ and $a_{i, j}=0$ if $i \neq j$. We put

$$
\mathbf{g}_{\sigma}=\left(g_{\sigma}, \varphi_{D, r}^{-n_{\sigma}}, \sigma\right) \in G .
$$

Then $\mathbf{g}_{\sigma}$ stabilizes each component in (1.5). We choose elements $\alpha_{r}, \beta_{r}, \gamma_{r} \in K^{\mathrm{ac}}$ such that

$$
\begin{aligned}
& \alpha_{r}^{p^{e}+1}=-\varphi_{r}^{\prime}, \quad \beta_{r}^{p^{2 e}}+\beta_{r}=-\alpha_{r}^{-1}, \quad \gamma_{r}^{p^{m}}-\gamma_{r}=\beta_{r}^{p^{e}+1}+\varepsilon_{0}, \\
& \alpha_{r}^{-1} \eta_{r}^{\frac{p^{e}}{p^{e}+1}} \equiv 1, \quad \beta_{r}^{-1} \theta_{r}^{p^{e}} \eta_{r}^{-\frac{p^{e}}{p^{e}+1}} \equiv 1, \quad \gamma_{r}^{-1} \sum_{i=0}^{\frac{f}{m}-1}\left(\lambda_{r} \eta_{r}^{-1}\right)^{p^{i m}} \equiv 1 \quad \bmod >0 .
\end{aligned}
$$

For $\sigma \in W_{E_{r}}$, we set

$$
\begin{aligned}
& a_{r, \sigma}=\frac{\sigma\left(\alpha_{r}\right)}{\alpha_{r}}, \quad b_{r, \sigma}=a_{r, \sigma} \sigma\left(\beta_{r}\right)-\beta_{r}, \\
& c_{r, \sigma}=\sigma\left(\gamma_{r}\right)-\gamma_{r}+\sum_{i=0}^{\frac{e}{m}-1}\left(b_{r, \sigma}^{p^{e}}\left(\beta_{r}+b_{r, \sigma}\right)\right)^{p^{i m}} .
\end{aligned}
$$

Then we have $a_{r, \sigma}, b_{r, \sigma}, c_{r, \sigma} \in \mathcal{O}_{\mathbf{C}}$ and

$$
\begin{aligned}
a_{r, \sigma} & \equiv\left(\frac{\sigma\left(\eta_{r}^{\frac{1}{p^{e}+1}}\right)}{\eta_{r}^{\frac{1}{p^{e}+1}}}\right)^{p^{e}}, \quad b_{r, \sigma} \equiv\left(\frac{\sigma\left(\theta_{r}\right)-\theta_{r}}{\eta_{r}^{\frac{1}{p^{e}+1}}}\right)^{p^{e}}, \\
c_{r, \sigma} & \equiv \sum_{i=0}^{\frac{f}{m}-1}\left(\frac{\sigma\left(\lambda_{r}\right)-\lambda_{r}}{\eta_{r}}\right)^{p^{i m}}-\sum_{i=0}^{\frac{e}{m}-1}\left(\frac{\left(\sigma\left(\theta_{r}\right)-\theta_{r}\right) \sigma\left(\theta_{r}\right)^{p^{e}}}{\eta_{r}}\right)^{p^{i m}} \quad \bmod _{>} 0
\end{aligned}
$$

by (2.1) and (3.16). Let

$$
Q=\left\{g(a, b, c) \mid a, b, c \in k^{\mathrm{ac}}, a^{p^{e}+1}=1, b^{p^{2 e}}+b=0, c^{p^{m}}-c+b^{p^{e}+1}=0\right\}
$$

be the group whose multiplication is given by

$$
g\left(a_{1}, b_{1}, c_{1}\right) \cdot g\left(a_{2}, b_{2}, c_{2}\right)=g\left(a_{1} a_{2}, a_{1} b_{2}+b_{1}, c_{1}+c_{2}+\sum_{i=0}^{\frac{e}{m}-1}\left(a_{1} b_{1}^{p^{e}} b_{2}\right)^{p^{i m}}\right) .
$$


Let $Q \rtimes \mathbb{Z}$ be the semidirect product, where $l \in \mathbb{Z}$ acts on $Q$ by $g(a, b, c) \mapsto$ $g\left(a^{q^{-l}}, b^{q^{-l}}, c^{q^{-l}}\right)$. Let $(g(a, b, c), l) \in Q \rtimes \mathbb{Z}$ act on $\overline{\mathfrak{X}}_{r}$ by

$\left(\boldsymbol{z}, \boldsymbol{y},\left(\boldsymbol{y}_{i}\right)_{1 \leq i \leq n-2}\right) \mapsto\left(\left(z+\sum_{i=0}^{\frac{e}{m}-1}(b \boldsymbol{y})^{p^{i m}}+c\right)^{q^{l}},\left(a\left(\boldsymbol{y}+b^{p^{e}}\right)\right)^{q^{l}},\left(a^{\frac{p^{e}+1}{2}} \boldsymbol{y}_{i}^{q^{l}}\right)_{1 \leq i \leq n-2}\right)$.

We have the surjective homomorphism

$$
\Theta_{r}: W_{E_{r}} \rightarrow Q \rtimes \mathbb{Z} ; \sigma \mapsto\left(g\left(\bar{a}_{r, \sigma}, \bar{b}_{r, \sigma}, \bar{c}_{r, \sigma}\right), n_{\sigma}\right) .
$$

Proposition 3.4 Let $\sigma \in W_{E_{r}}$. Then, $\mathbf{g}_{\sigma} \in G$ stabilizes $\mathcal{X}_{r}$, and induces the automorphism of $\overline{\mathfrak{X}}_{r}$ given by $\Theta_{r}(\sigma)$.

Proof Let $P \in \mathcal{X}_{r}(\mathbf{C})$. We have

$$
\begin{aligned}
\boldsymbol{S}\left(P \mathbf{g}_{\sigma}\right) & =f_{0}\left(\boldsymbol{X}\left(P \mathbf{g}_{\sigma}\right)\right)-f_{0}\left(\boldsymbol{\xi}_{r}^{0}\right) \\
& =f_{0}\left(\boldsymbol{X}\left(P \mathbf{g}_{\sigma}\right)\right)-f_{0}\left(\boldsymbol{X}\left(P\left(1, \varphi_{D, r}^{-n_{\sigma}}, \sigma\right)\right)\right)+\sigma^{-1}\left(f_{0}(\boldsymbol{X}(P))\right)-f_{0}\left(\boldsymbol{\xi}_{r}^{0}\right) \\
& \equiv \Delta_{g_{\sigma}}\left(\boldsymbol{X}\left(P\left(1, \varphi_{D, r}^{-n_{\sigma}}, \sigma\right)\right)\right)+\sigma^{-1}\left(\boldsymbol{S}(P)+f_{0}\left(\boldsymbol{\xi}_{r}^{0}\right)\right)-f_{0}\left(\boldsymbol{\xi}_{r}^{0}\right) \\
& \equiv \sigma^{-1}(\boldsymbol{S}(P))+f_{0}\left(\sigma^{-1}\left(\boldsymbol{\xi}_{r}^{0}\right)\right)-f_{0}\left(\boldsymbol{\xi}_{r}^{0}\right) \quad \bmod _{>} \frac{1}{n}
\end{aligned}
$$

by (2.5) and (3.12). We have

$$
f_{0}\left(\sigma^{-1}\left(\xi_{r}^{0}\right)\right)-f_{0}\left(\xi_{r}^{0}\right) \equiv n^{\prime}\left(\sigma^{-1}\left(\lambda_{r}\right)-\lambda_{r}\right) \quad \bmod _{>} \frac{1}{n}
$$

We put $s_{i}(\boldsymbol{X})=\left(\boldsymbol{X}_{i} / \boldsymbol{X}_{i+1}\right)^{q^{i}(q-1)}$ for $1 \leq i \leq n-1$. We have

$$
\begin{aligned}
& s_{n-1}\left(\boldsymbol{\xi}_{r}^{0}\right) \boldsymbol{Y}_{n-1}\left(P \mathbf{g}_{\sigma}\right)=s_{n-1}\left(\boldsymbol{X}\left(P \mathbf{g}_{\sigma}\right)\right)-s_{n-1}\left(\boldsymbol{\xi}_{r}^{0}\right) \\
& =s_{n-1}\left(\boldsymbol{X}\left(P \mathbf{g}_{\sigma}\right)\right)-s_{n-1}\left(\boldsymbol{X}\left(P\left(1, \varphi_{D, r}^{-n_{\sigma}}, \sigma\right)\right)\right)+\sigma^{-1}\left(s_{n-1}(\boldsymbol{X}(P))\right)-s_{n-1}\left(\boldsymbol{\xi}_{r}^{0}\right) \\
& \equiv \sigma^{-1}\left(s_{n-1}\left(\xi_{r}^{0}\right) \boldsymbol{Y}_{n-1}(P)\right)+\sigma^{-1}\left(s_{n-1}\left(\xi_{r}^{0}\right)\right)-s_{n-1}\left(\xi_{r}^{0}\right) \quad \bmod _{>} \frac{q-1}{n}+\frac{1}{n p^{e}}
\end{aligned}
$$

by (2.6) and (3.12). Hence, we have

$$
\boldsymbol{Y}_{n-1}\left(P \mathbf{g}_{\sigma}\right) \equiv \sigma^{-1}\left(\boldsymbol{Y}_{n-1}(P)\right)+\sigma^{-1}\left(\boldsymbol{\theta}_{r}\right)-\boldsymbol{\theta}_{r} \quad \bmod >\frac{1}{n p^{e}} .
$$

We put $\boldsymbol{\theta}_{r, \sigma}=\sigma\left(\boldsymbol{\theta}_{r}\right)-\boldsymbol{\theta}_{r}$ and $\lambda_{r, \sigma}=\sigma\left(\boldsymbol{\lambda}_{r}\right)-\boldsymbol{\lambda}_{r}$. We have

$$
\sigma\left(z\left(P \mathbf{g}_{\sigma}\right)\right)=\sigma\left(\sum_{i=0}^{\frac{e}{m}-1}\left(\frac{\boldsymbol{\theta}_{r}^{p^{e}} \boldsymbol{Y}_{n-1}\left(P \mathbf{g}_{\sigma}\right)}{\eta_{r}}\right)^{p^{i m}}-\frac{1}{n^{\prime}} \sum_{i=0}^{\frac{f}{m}-1}\left(\frac{\boldsymbol{S}\left(P \mathbf{g}_{\sigma}\right)}{\eta_{r}}\right)^{p^{i m}}\right)
$$




$$
\begin{aligned}
& \equiv \boldsymbol{z}(P)+\sum_{i=0}^{\frac{e}{m}-1}\left(\frac{\boldsymbol{\theta}_{r, \sigma}^{p^{e}} \boldsymbol{Y}_{n-1}(P)-\sigma\left(\boldsymbol{\theta}_{r}^{p^{e}}\right) \boldsymbol{\theta}_{r, \sigma}}{\boldsymbol{\eta}_{r}}\right)^{p^{i m}}+\sum_{i=0}^{\frac{f}{m}-1}\left(\frac{\lambda_{r, \sigma}}{\boldsymbol{\eta}_{r}}\right)^{p^{i m}} \\
& \equiv \boldsymbol{z}(P)+\sum_{i=0}^{\frac{e}{m}-1}\left(b_{r, \sigma} \boldsymbol{y}(P)\right)^{p^{i m}}+c_{r, \sigma} \quad \bmod _{>} 0
\end{aligned}
$$

by (2.7), (3.17), (3.20), (3.21), (3.22) and (3.23). We see also that

$$
\sigma\left(\frac{\boldsymbol{Y}_{n-1}\left(P \mathbf{g}_{\sigma}\right)}{\boldsymbol{\eta}_{r}^{1 /\left(p^{e}+1\right)}}\right) \equiv a_{r, \sigma}^{-p^{e}}\left(\boldsymbol{y}-b_{r, \sigma}^{\frac{1}{p^{e}}}\right) \equiv a_{r, \sigma}\left(\boldsymbol{y}+b_{r, \sigma}^{p^{e}}\right) \quad \bmod >0
$$

by (3.17) and (3.23). By the same argument using (3.11), we have

$$
\boldsymbol{Y}_{i}\left(P \mathbf{g}_{\sigma}\right) \equiv \sigma^{-1}\left(\boldsymbol{Y}_{i}(P)\right) \quad \bmod >\frac{1}{2 n}
$$

for $1 \leq i \leq n-1$. This implies

$$
\boldsymbol{y}_{i}\left(P \mathbf{g}_{\sigma}\right) \equiv \frac{\sigma^{-1}\left(\boldsymbol{\eta}_{r}^{1 / 2}\right)}{\boldsymbol{\eta}_{r}^{1 / 2}} \sigma^{-1}\left(\boldsymbol{y}_{i}(P)\right) \equiv a_{r, \sigma}^{\left(p^{e}+1\right) / 2} \boldsymbol{y}_{i}(P)^{q^{n_{\sigma}}} \quad \bmod _{>} 0
$$

for $1 \leq i \leq n-1$ by (3.17).

Stabilizer We put $n_{1}=\operatorname{gcd}\left(n, p^{m}-1\right)$. We put

$$
\varphi_{r}^{\prime \prime}=\varphi_{r}^{n_{1}} \quad \text { and } \quad F_{r}=K\left(\varphi_{r}^{\prime \prime}\right)
$$

Let $\sigma \in W_{F_{r}}$. We put

$$
\zeta_{\sigma}=\frac{\sigma^{-1}\left(\varphi_{r}^{\prime}\right)}{\varphi_{r}^{\prime}}
$$

Let $\zeta_{\sigma}^{1 / p^{e}}$ be the $p^{e}$-th root of $\zeta_{\sigma}$ in $\mu_{p^{m}-1}(K)$. We put

$$
\varphi_{r, \sigma}=\zeta_{\sigma}^{1 / p^{e}} \varphi_{r}
$$

Let $\mathcal{G}_{r, \sigma}$ be the one-dimensional formal $\mathcal{O}_{L_{r}}$-module over $\mathcal{O}_{\widehat{L}_{r}^{\text {ur }}}$ defined similarly to $\mathcal{G}_{r}$ changing $\varphi_{r}$ by $\varphi_{r, \sigma}$. We take a compatible system $\left\{t_{r, j, \sigma}\right\}_{j \geq 1}$ in $\mathbf{C}$ such that

$$
\frac{\sigma^{-1}\left(t_{r, 1}\right)}{t_{r, 1, \sigma}} \equiv 1 \quad \bmod _{>} 0, \quad\left[\varphi_{r, \sigma}\right]_{\mathcal{G}_{r, \sigma}}\left(t_{r, 1, \sigma}\right)=0, \quad\left[\varphi_{r, \sigma}\right]_{\mathcal{G}_{r, \sigma}}\left(t_{r, j, \sigma}\right)=t_{r, j-1, \sigma}
$$

for $j \geq 2$. We construct $\xi_{r, \sigma}$ as in Lemma 2.1 using $\left\{t_{r, j, \sigma}\right\}_{j \geq 1}$. Then $\xi_{r, \sigma}$ has CM by $L_{r}$. 
Lemma 3.5 For $\sigma \in W_{F_{r}}$, we have

$$
\begin{aligned}
\frac{\sigma^{-1}\left(\xi_{r, i}\right)}{\xi_{r, \sigma, i}} & \equiv 1 \quad \bmod _{\geq} \frac{1}{q^{i-1} p^{e-1}(p-1)} \text { for } 1 \leq i \leq n \\
\sigma^{-1}\left(\boldsymbol{\theta}_{r}\right) & \equiv \boldsymbol{\theta}_{r} \quad \bmod _{\geq} \frac{1}{n\left(p^{e}+1\right)}
\end{aligned}
$$

Proof We have

$$
\frac{\sigma^{-1}\left(\varphi_{r}\right)}{\varphi_{r}} \equiv \zeta_{\sigma}^{1 / p^{e}} \quad \bmod _{\geq} \frac{1}{p^{e-1}(p-1)} .
$$

We obtain the claims by (3.24) and

$$
\begin{aligned}
& \left(\sigma^{-1}\left(\boldsymbol{\theta}_{r}\right)-\boldsymbol{\theta}_{r}\right)^{p^{2 e}}+\boldsymbol{\eta}_{r}^{p^{e}-1}\left(\sigma^{-1}\left(\boldsymbol{\theta}_{r}\right)-\boldsymbol{\theta}_{r}\right) \\
& \quad+\left(\mathbf{1}+\sigma^{-1}\left(\boldsymbol{\theta}_{r}\right)\right)\left(\sigma^{-1}\left(\boldsymbol{\eta}_{r}\right)^{p^{e}-1}-\boldsymbol{\eta}_{r}^{p^{e}-1}\right)=\mathbf{0},
\end{aligned}
$$

which follows from (2.1).

We define $j_{r}: W_{F_{r}} \rightarrow L_{r}^{\times} \backslash\left(G L_{n}(K) \times D^{\times}\right)$as follows:

Let $\sigma \in W_{F_{r}}$. Since $\xi_{r, \sigma}$ has CM by $L_{r}$, there exists $(g, d) \in G L_{n}(K) \times$ $D^{\times}$uniquely up to left multiplication by $L_{r}^{\times}$such that $(g, d, 1) \in G^{0}$ and $\xi_{r, \sigma}(g, d, 1)=\xi_{r}$ by Lemma 1.6. We put $j_{r}(\sigma)=L_{r}^{\times}\left(g, \varphi_{D, r}^{-n_{\sigma}} d\right)$.

For $\sigma \in W_{L_{r}}$, we put $a_{\sigma}=\operatorname{Art}_{L_{r}}^{-1}(\sigma) \in L_{r}^{\times}$and $u_{\sigma}=a_{\sigma} \varphi_{r}^{-n_{\sigma}} \in \mathcal{O}_{L_{r}}^{\times}$.

Lemma 3.6 For $\sigma \in W_{L_{r}}$, we have $j_{r}(\sigma)=L_{r}^{\times}\left(1, a_{\sigma}^{-1}\right)$.

Proof This follows from [5, Lemma 3.1.3]. Note that our action of $W_{K}$ is inverse to that in [5].

We put

$$
\mathcal{S}_{r}=\left\{(g, d, \sigma) \in G \mid \sigma \in W_{F_{r}}, j_{r}(\sigma)=L_{r}^{\times}(g, d)\right\} .
$$

Lemma 3.7 The action of $\mathcal{S}_{r}$ on $\mathcal{M}_{\infty, \bar{\eta}}^{(0)}$ stabilizes $\mathcal{X}_{r}$, and induces the action on $\overline{\mathfrak{X}}_{r}$.

Proof We take an element of $\mathcal{S}_{r}$, and write it as $\left(g, \varphi_{D, r}^{-n_{\sigma}} d, \sigma\right)$, where $(g, d, 1) \in G^{0}$ and $\sigma \in W_{F_{r}}$. Since $\xi_{r, \sigma}(g, d, 1)=\xi_{r}$, we have $(g, d) \in\left(\varphi_{M, r}, \varphi_{D, r}\right)^{l}\left(\mathfrak{I}^{\times} \times \mathcal{O}_{D}^{\times}\right)$by Lemma 3.1 and Lemma 3.5.

To show the claims, we may assume that $(g, d) \in \mathfrak{I}^{\times} \times \mathcal{O}_{D}^{\times}$by Proposition $3.2(1)$. We write $g=\left(a_{i, j}\right)_{1 \leq i, j \leq n} \in \mathfrak{I}^{\times}$and $a_{i, j}=\sum_{l=0}^{\infty} a_{i, j}^{(l)} \varpi_{r}^{l}$ with $a_{i, j}^{(l)} \in \mu_{q-1}(K) \cup\{0\}$, and $d^{-1}=\sum_{i=0}^{\infty} d_{i} \varphi_{D, r}^{i}$ with $d_{i} \in \mu_{q^{n}-1}\left(K_{n}\right) \cup\{0\}$. For $1 \leq i \leq n-1$, we have

$$
\frac{a_{i, i}^{(0)}}{a_{i+1, i+1}^{(0)}}=d_{0}^{q-1}
$$


by $\xi_{r, \sigma}(g, d, 1)=\xi_{r}$ using (3.10), (3.11), $\xi_{r, \sigma, i}=\xi_{r, \sigma, i+1}^{q}$ and $\xi_{r, i}=\xi_{r, i+1}^{q}$. The condition on the first line in (2.2) is equivalent to

$$
v\left(\frac{\boldsymbol{X}_{i}}{\boldsymbol{X}_{i+1}}-\left(\frac{\boldsymbol{X}_{n-1}}{\boldsymbol{X}_{n}}\right)^{q^{n-1-i}}\right) \geq \frac{3}{2 n q^{i}} \text { for } 1 \leq i \leq n-2 .
$$

We see that the condition (3.26) is stable under the action of $\left(g, \varphi_{D, r}^{-n_{\sigma}} d, \sigma\right)$ using (3.10) and (3.11), because $a_{i, i}^{(0)} / a_{i+1, i+1}^{(0)}$ is independent of $i$ by (3.25). We see that the condition on the second line in (2.2) is stable under the action of $\left(g, \varphi_{D, r}^{-n_{\sigma}} d, \sigma\right)$ by Lemma 3.5 using (3.10) and (3.11).

The group $\mathcal{S}_{r}$ normalizes $i_{\xi_{r}}\left(L_{r}^{\times}\right) \cdot\left(U_{\mathfrak{I}}^{1} \times U_{D}^{1}\right)^{1}$ by Proposition 3.3. We put

$$
H_{r}=\left(U_{\mathfrak{I}}^{1} \times U_{D}^{1}\right)^{1} \cdot \mathcal{S}_{r} \subset G .
$$

Then $H_{r}$ acts on $\overline{\mathfrak{X}}_{r}$ by Lemma 3.7 and the proof of Proposition 3.3.

Proposition 3.8 The subgroup $H_{r} \subset G^{0}$ is the stabilizer of $\mathcal{X}_{r}$ in $\mathcal{M}_{\infty, \bar{\eta}}^{(0)}$.

Proof Assume that $\left(g, \varphi_{D, r}^{-n_{\sigma}} d, \sigma\right) \in G^{0}$ stabilizes $\mathcal{X}_{r}$. It suffices to show that

$$
\left(g, \varphi_{D, r}^{-n_{\sigma}} d, \sigma\right) \in H_{r}
$$

By Lemma 3.1, we have $(g, d) \in\left(\varphi_{M, r}, \varphi_{D, r}\right)^{l}\left(\mathfrak{I}^{\times} \times \mathcal{O}_{D}^{\times}\right)$. Hence, we may assume that $(g, d) \in \mathfrak{I}^{\times} \times \mathcal{O}_{D}^{\times}$by Proposition $3.2(1)$.

First, we show that $\sigma \in W_{F_{r}}$. We write $g=\left(a_{i, j}\right)_{1 \leq i, j \leq n} \in \mathfrak{I}^{\times}, a_{i, j}=$ $\sum_{l=0}^{\infty} a_{i, j}^{(l)} \varpi_{r}^{l}$ and $d^{-1}=\sum_{i=0}^{\infty} d_{i} \varphi_{D, r}^{i}$ as in the proof of Lemma 3.7. Since $\left(g, \varphi_{D, r}^{-n_{\sigma}} d, \sigma\right)$ stabilizes $\mathcal{X}_{r}$, we have

$$
\begin{array}{r}
\frac{a_{i, i}^{(0)}}{a_{i+1, i+1}^{(0)}}=d_{0}^{q-1} \quad \text { for } 1 \leq i \leq n-1, \\
\frac{a_{n, n}^{(0)} d_{0} \sigma^{-1}\left(\xi_{r, n}^{0}\right)}{\xi_{r, n}^{0}} \equiv 1 \quad \bmod _{\geq} \frac{1}{n q^{n-1}\left(p^{e}+1\right)}
\end{array}
$$

by (2.2), (3.10), (3.11) and $\xi_{r, i}=\xi_{r, i+1}^{q}$. By taking the $p^{e} q^{n-1}(q-1)$-st power of (3.28), we see that

$$
d_{0}^{p^{e} q^{n-1}(q-1)} \frac{\sigma^{-1}\left(\varphi_{r}^{\prime}\right)}{\varphi_{r}^{\prime}} \equiv\left(\frac{1+\theta_{r}}{1+\sigma^{-1}\left(\theta_{r}\right)}\right)^{p^{e}(q-1)} \quad \bmod _{\geq} \frac{p^{e}}{n\left(p^{e}+1\right)} .
$$

This implies that the left hand side of (3.29) is equal to 1. Hence we have $\sigma^{-1}\left(\varphi_{r}^{\prime}\right) / \varphi_{r}^{\prime} \in \mu_{q-1}(K)$ and $\sigma^{-1}\left(\theta_{r}\right) \equiv \theta_{r} \bmod _{\geq} 1 /\left(n\left(p^{e}+1\right)\right)$, since $d_{0}^{q-1} \in$ $\mu_{q-1}(K)$ by (3.27). These happen only if $\sigma \in W_{F_{r}}$ by the proof of Lemma 3.5 and 
$\mu_{p^{e}-1}\left(K^{\text {ur }}\right) \cap \mu_{q-1}(K)=\mu_{p^{m}-1}(K)$. Since $\sigma \in W_{F_{r}}$, we may assume that $\sigma=1$ by Lemma 3.7. Then $(g, d, 1) \in H_{r}$ by Proposition 3.3.

\section{Artin-Schreier variety}

\subsection{Tate conjecture}

Let $m$ be a positive integer such that $\mathbb{F}_{p^{m}} \subset \mathbb{F}_{q}$. Let $N$ be a positive even integer. We put $n_{0}=N / 2$. We consider the affine smooth variety $X_{N, \mathbb{F}_{q}}$ over $\mathbb{F}_{q}$ defined by

$$
z^{p^{m}}-z=\sum_{i=1}^{n_{0}} u_{2 i-1} u_{2 i} \text { in } \mathbb{A}_{\mathbb{F}_{q}}^{N+1}
$$

Let $X_{N}$ be the base change of $X_{N, \mathbb{F}_{q}}$ to $\overline{\mathbb{F}}_{q}$. For an integer $i \geq 0$, we simply write $\mathbb{A}^{i}$ for the affine space $\mathbb{A}_{\overline{\mathbb{F}}_{q}}^{i}$.

Remark 4.1 Let $Q\left(y_{1}, \ldots, y_{N}\right)$ be any non-degenerate quadratic form on $\mathbb{A}^{N}$. Then the affine smooth variety over $\overline{\mathbb{F}}_{q}$ defined by

$$
z^{p^{m}}-z=Q\left(y_{1}, \ldots, y_{N}\right) \text { in } \mathbb{A}^{N+1}
$$

is isomorphic to $X_{N}$ by [22, XII, Proposition 1.2].

For each $\zeta \in \mathbb{F}_{p^{m}}^{\times}$, we consider the homomorphism

$$
p_{\zeta}: \mathbb{F}_{p^{m}} \rightarrow \mathbb{F}_{p} ; x \mapsto \operatorname{Tr}_{\mathbb{F}_{p} / \mathbb{F}_{p}}\left(\zeta^{-1} x\right)
$$

Then, we consider the quotient $X_{N, \zeta}=X_{N} / \operatorname{ker} p_{\zeta}$. Note that the quotient $X_{N, \zeta}$ depends only on the class $[\zeta] \in \mathbb{F}_{p^{m}}^{\times} / \mathbb{F}_{p}^{\times}$of $\zeta$. The variety $X_{N, \zeta}$ has the defining equation

$$
\zeta\left(z_{\zeta}^{p}-z_{\zeta}\right)=\sum_{i=1}^{n_{0}} u_{2 i-1} u_{2 i} \quad \text { in } \mathbb{A}^{N+1}
$$

where the relation between $z$ and $z_{\zeta}$ is given by $z_{\zeta}=\sum_{i=0}^{m-1}\left(\zeta^{-1} z\right)^{p^{i}}$. Let $\ell \neq p$ be a prime number. For a topological abelian group $A$, let $A^{\vee}$ denote the set of the smooth characters $A \rightarrow \overline{\mathbb{Q}}_{\ell}^{\times}$. Let $\mathcal{L}_{\psi}$ be the Artin-Schreier $\overline{\mathbb{Q}}_{\ell}$-sheaf on $\mathbb{A}^{1}$ associated to $\psi \in \mathbb{F}_{p^{m}}^{\vee}$, which is $\mathfrak{F}(\psi)$ in the notation of [6, Sommes trig. 1.8 (i)]. For a polynomial $f \in \overline{\mathbb{F}}_{q}\left[x_{1}, \ldots, x_{l}\right]$, let $\mathcal{L}_{\psi}(f)$ denote the pullback of $\mathcal{L}_{\psi}$ under $f: \mathbb{A}^{l} \rightarrow \mathbb{A}^{1}$.

Lemma 4.2 We have an isomorphism

$$
\bigoplus_{[\zeta] \in \mathbb{F}_{p^{m}}^{\times} / \mathbb{F}_{p}^{\times}} H^{N}\left(X_{N, \zeta}, \overline{\mathbb{Q}}_{\ell}\right) \simeq H^{N}\left(X_{N}, \overline{\mathbb{Q}}_{\ell}\right)
$$


induced by the pullbacks and $\operatorname{dim} H^{N}\left(X_{N, \zeta}, \overline{\mathbb{Q}}_{\ell}\right)=p-1$.

Proof For $\psi \in \mathbb{F}_{p^{m}}^{\vee} \backslash\{1\}$, we have

$$
H_{\mathrm{c}}^{i}\left(\mathbb{A}^{2}, \mathcal{L}_{\psi}(x y)\right)= \begin{cases}\overline{\mathbb{Q}}_{\ell}(-1) & \text { if } i=2 \\ 0 & \text { otherwise }\end{cases}
$$

by [19, Proposition 1.2.2.2] as in the proof of [16, Lemma 2.1]. Hence, by the Künneth formula, we have isomorphisms

$$
H_{\mathrm{c}}^{N}\left(X_{N}, \overline{\mathbb{Q}}_{\ell}\right) \simeq \bigoplus_{\psi \in \mathbb{F}_{p^{m}}^{\vee} \backslash\{1\}} H_{\mathrm{c}}^{N}\left(\mathbb{A}^{N}, \mathcal{L}_{\psi}\left(\sum_{i=1}^{n_{0}} u_{2 i-1} u_{2 i}\right)\right) \simeq \bigoplus_{\psi \in \mathbb{F}_{p^{m} \backslash\{1\}}^{\vee}} \psi
$$

as $\mathbb{F}_{p^{m}}$-representations. By Poincaré duality, we have an isomorphism

$$
H^{N}\left(X_{N}, \overline{\mathbb{Q}}_{\ell}\right) \simeq \bigoplus_{\psi \in \mathbb{F}_{p^{m}}^{\vee} \backslash\{1\}} \psi
$$

as $\mathbb{F}_{p^{m}}$-representations. Let $\psi^{\prime}: \mathbb{F}_{p} \hookrightarrow \overline{\mathbb{Q}}_{\ell}^{\times}$be any non-trivial character. Then, for each $\psi \in \mathbb{F}_{p^{m}}^{\vee} \backslash\{1\}$, there exists a unique element $\zeta \in \mathbb{F}_{p^{m}}^{\times}$such that $\psi=\psi^{\prime} \circ p_{\zeta}$. Hence, we know that

$$
H^{N}\left(X_{N, \zeta}, \overline{\mathbb{Q}}_{\ell}\right)\left[\psi^{\prime}\right]=H^{N}\left(X_{N}, \overline{\mathbb{Q}}_{\ell}\right)[\psi] \simeq \psi
$$

as $\mathbb{F}_{p^{m}}$-representations. Therefore, the required assertion follows.

Consider the fibration

$$
\pi_{\zeta}: X_{N, \zeta} \rightarrow \mathbb{A}^{n_{0}} ;\left(z_{\zeta},\left(u_{i}\right)_{1 \leq i \leq N}\right) \mapsto\left(\left(u_{2 i}\right)_{1 \leq i \leq n_{0}}\right)
$$

Let $\mathbf{0}$ denote the origin of $\mathbb{A}^{n_{0}}$. The inverse image $\pi_{\zeta}^{-1}(\mathbf{0})$ has $p$ connected components. For $a \in \mathbb{F}_{p}$, we define $Z_{\zeta}^{a}$ to be the connected component of $\pi_{\zeta}^{-1}(\mathbf{0})$ defined by $z_{\zeta}=a$. We know that each $Z_{\zeta}^{a}$ is isomorphic to the affine space of dimension $n_{0}$. Let

$$
\mathrm{cl}: C H_{n_{0}}\left(X_{N, \zeta}\right) \rightarrow H^{N}\left(X_{N, \zeta}, \overline{\mathbb{Q}}_{\ell}\left(n_{0}\right)\right)
$$

be the cycle class map.

Lemma 4.3 (1) The fibration $\pi_{\zeta}: X_{N, \zeta} \rightarrow \mathbb{A}^{n_{0}}$ is an affine bundle over $\mathbb{A}^{n_{0}} \backslash\{\mathbf{0}\}$.

(2) The cohomology group $H^{N}\left(X_{N, \zeta}, \overline{\mathbb{Q}}_{\ell}\left(n_{0}\right)\right)$ is generated by the cycle classes $\operatorname{cl}\left(\left[Z_{\zeta}^{a}\right]\right)$ for $a \in \mathbb{F}_{p}$ with the relation $\sum_{a \in \mathbb{F}_{p}} \operatorname{cl}\left(\left[Z_{\zeta}^{a}\right]\right)=0$. 
Proof For $1 \leq i \leq n_{0}$, let $U_{i}$ be the open subscheme of $\mathbb{A}^{n_{0}}$ defined by the condition that the $i$-th coordinate is not zero. Then $\left\{U_{i}\right\}_{1 \leq i \leq n_{0}}$ is a covering of $\mathbb{A}^{n_{0}} \backslash\{\boldsymbol{0}\}$. We can see that $\pi_{\zeta}$ is a trivial affine bundle on each $U_{i}$ by (4.1). Hence the first claim follows.

We set $U=\pi_{\zeta}^{-1}\left(\mathbb{A}^{n_{0}} \backslash\{\boldsymbol{0}\}\right)$. We have the long exact sequence

$$
\begin{aligned}
H^{N-1}\left(U, \overline{\mathbb{Q}}_{\ell}\right) & \rightarrow H_{\pi_{\zeta}^{-1}(\mathbf{0})}^{N}\left(X_{N, \zeta}, \overline{\mathbb{Q}}_{\ell}\right) \simeq \overline{\mathbb{Q}}_{\ell}\left(-n_{0}\right)^{\oplus p} \\
& \rightarrow H^{N}\left(X_{N, \zeta}, \overline{\mathbb{Q}}_{\ell}\right) \rightarrow H^{N}\left(U, \overline{\mathbb{Q}}_{\ell}\right)
\end{aligned}
$$

and $H^{N}\left(U, \overline{\mathbb{Q}}_{\ell}\right) \simeq H^{N}\left(\mathbb{A}^{n_{0}} \backslash\{\mathbf{0}\}, \overline{\mathbb{Q}}_{\ell}\right)=0$, which follows from the first claim. Therefore, $H^{N}\left(X_{N, \zeta}, \overline{\mathbb{Q}}_{\ell}\left(n_{0}\right)\right)$ is generated by the cycle classes $\operatorname{cl}\left(\left[Z_{\zeta}^{a}\right]\right)$ for $a \in$ $\mathbb{F}_{p}$. On the other hand, we have $\sum_{a \in \mathbb{F}_{p}} \operatorname{cl}\left(\left[Z_{\zeta}^{a}\right]\right)=0$, since $\sum_{a \in \mathbb{F}_{p}}\left[Z_{\zeta}^{a}\right]=0$ in $C H_{n_{0}}\left(X_{N, \zeta}\right)$. Since $\operatorname{dim} H^{N}\left(X_{N, \zeta}, \overline{\mathbb{Q}}_{\ell}\left(n_{0}\right)\right)=p-1$ by Lemma 4.2, we obtain the claim.

Corollary 4.4 The Tate conjecture in $[18,7.13]$ holds for the variety $X_{N, \mathbb{F}_{q}}$.

Proof By Lemma 4.2, Lemma 4.3 and the commutativity of cycle maps and pullbacks under $X_{N} \rightarrow X_{N, \zeta}$, we have $\left[18,7.13\right.$ Conjecture (A), (B)] for $X_{N, \mathbb{F}_{q}}$ and the equality

$$
H^{N}\left(X_{N}, \overline{\mathbb{Q}}_{\ell}\left(n_{0}\right)\right)^{\mathrm{Gal}\left(\overline{\mathbb{F}}_{q} / \mathbb{F}_{q}\right)}=H^{N}\left(X_{N}, \overline{\mathbb{Q}}_{\ell}\left(n_{0}\right)\right) .
$$

Then the $q$-th geometric Frobenius in $\operatorname{Gal}\left(\overline{\mathbb{F}}_{q} / \mathbb{F}_{q}\right)$ acts on $H^{N}\left(X_{N}, \overline{\mathbb{Q}}_{\ell}\right)$ by $q^{n_{0}}$. Hence $\left[18,7.13\right.$ Conjecture (C)] for $X_{N, \mathbb{F}_{q}}$ also follows.

\subsection{Action on cohomology}

In this section, we assume that $p=2$. Let $n \geq 4$ be an even integer. Let $m=\operatorname{gcd}(e, f)$ as in Sect. 2.2. We consider the affine smooth variety $X$ of dimension $n-2$ defined by

$$
z^{2^{m}}-z=\sum_{1 \leq i \leq j \leq n-2} y_{i} y_{j} \text { in } \mathbb{A}^{n-1} \text {. }
$$

We take $\zeta_{3} \in \overline{\mathbb{F}}_{q} \backslash\{1\}$ such that $\zeta_{3}^{3}=1$. Then, we define $u_{1}, \ldots, u_{n-2}$ by

$$
\begin{aligned}
& u_{4 i+1}=\zeta_{3} y_{4 i+1}+\zeta_{3}^{-1} y_{4 i+2}+\sum_{j=4 i+3}^{n-2} y_{j}, \quad u_{4 i+2}=\zeta_{3}^{-1} y_{4 i+1}+\zeta_{3} y_{4 i+2}+\sum_{j=4 i+3}^{n-2} y_{j}, \\
& u_{4 i+3}=y_{4 i+3}+\sum_{j=4 i+5}^{n-2} y_{j}, \quad u_{4 i+4}=y_{4 i+4}+\sum_{j=4 i+5}^{n-2} y_{j} .
\end{aligned}
$$


Then the variety $X$ is isomorphic to the affine variety $X_{n-2}$ defined by

$$
z^{2^{m}}-z=\sum_{i=1}^{n_{0}} u_{2 i-1} u_{2 i} \text { in } \mathbb{A}^{n-1},
$$

where $n_{0}=(n-2) / 2$. For $\zeta \in \mathbb{F}_{2^{m}}^{\times}$, we simply write $X_{\zeta}$ for the variety $X_{n-2, \zeta}$, which is defined in Sect. 4.1 where $N=n-2$. Recall that $X_{\zeta}$ has the defining equation

$$
\zeta\left(z_{\zeta}^{2}-z_{\zeta}\right)=\sum_{i=1}^{n_{0}} u_{2 i-1} u_{2 i} \text { in } \mathbb{A}^{n-1} .
$$

For $a \in \mathbb{F}_{2}$, we consider the other $n_{0}$-dimensional cycle $Z_{\zeta}^{\prime a}$ in $X_{\zeta}$ defined by

$$
\begin{aligned}
& u_{1}=0, \quad u_{4 i-1}=u_{4 i+2}, \quad u_{4 i}=u_{4 i+1} \quad \text { for } 1 \leq i \leq\left[\left(n_{0}-1\right) / 2\right], \\
& u_{n-3}=u_{n-2}+1 \text { if } e=1, \quad z_{\zeta}=a+\varepsilon_{1} u_{n-2},
\end{aligned}
$$

where $\varepsilon_{1}$ is defined at (3.2).

Proposition 4.5 For $\zeta \in \mathbb{F}_{2^{m}}^{\times}$and $a \in \mathbb{F}_{2}$, we have

$$
\left[Z_{\zeta}^{a}\right]=(-1)^{n_{0}}\left[Z_{\zeta}^{\prime a}\right] \text { in } C H_{n_{0}}\left(X_{\zeta}\right)
$$

Proof We show that $\left[Z_{\zeta}^{a}\right]-(-1)^{n_{0}}\left[Z_{\zeta}^{\prime a}\right]$ is rationally equivalent to zero. For $1 \leq i \leq$ $\left[\left(n_{0}+1\right) / 2\right]$, let $X_{\zeta, i}$ be the $\left(n_{0}+1\right)$-dimensional closed subvariety of $X_{\zeta}$ defined by

$$
\begin{aligned}
& u_{4 j}=0 \text { for } 1 \leq j \leq\left[n_{0} / 2\right], \\
& u_{4 j-3}=0 \text { for } 1 \leq j \leq i-1, \quad u_{4 j-2}=0 \text { for } i+1 \leq j \leq\left[\left(n_{0}+1\right) / 2\right]
\end{aligned}
$$

and let $Z_{\zeta, i}^{a}$ be the $n_{0}$-dimensional cycle on $X_{\zeta, i}$ defined by $u_{4 i-3}=0$ and $z_{\zeta}=a$. We put $Z_{\zeta, 0}^{a}=Z_{\zeta}^{a}$. Then we have

$$
\operatorname{div}\left(z_{\zeta}-a\right)=\left[Z_{\zeta, i-1}^{a}\right]+\left[Z_{\zeta, i}^{a}\right]
$$

in $C H_{n_{0}}\left(X_{\zeta, i}\right)$ for $1 \leq i \leq\left[\left(n_{0}+1\right) / 2\right]$, since we have $\zeta\left(z_{\zeta}^{2}-z_{\zeta}\right)=u_{4 i-3} u_{4 i-2}$ on $X_{\zeta, i}$. For $1 \leq i \leq\left[\left(n_{0}-1\right) / 2\right]$, let $X_{\zeta, i}^{\prime}$ be the $\left(n_{0}+1\right)$-dimensional closed subvariety of $X_{\zeta}$ defined by

$$
\begin{aligned}
& u_{1}=0, \quad u_{4 j-1}=u_{4 j+2}, \quad u_{4 j}=u_{4 j+1} \quad \text { for } 1 \leq j \leq i-1, \quad u_{4 i}=u_{4 i+1} \\
& u_{4 j}=0 \quad \text { for } i+1 \leq j \leq\left[n_{0} / 2\right], \quad u_{4 j+1}=0 \text { for } i+1 \leq j \leq\left[\left(n_{0}-1\right) / 2\right]
\end{aligned}
$$

and let $Z_{\zeta, i}^{\prime a}$ be the $n_{0}$-dimensional cycle on $X_{\zeta, i}^{\prime}$ defined by $u_{4 i-1}=u_{4 i+2}$ and $z_{\zeta}=a$. We put $Z_{\zeta, 0}^{\prime a}=Z_{\zeta,\left[\left(n_{0}+1\right) / 2\right]}^{a}$. Then we have

$$
\operatorname{div}\left(z_{\zeta}-a\right)=\left[Z_{\zeta, i-1}^{a}\right]+\left[Z_{\zeta, i}^{\prime a}\right]
$$


in $C H_{n_{0}}\left(X_{\zeta, i}^{\prime}\right)$ for $1 \leq i \leq\left[\left(n_{0}-1\right) / 2\right]$, since we have

$$
\zeta\left(z_{\zeta}^{2}-z_{\zeta}\right)=u_{4 i}\left(u_{4 i-1}+u_{4 i+2}\right)
$$

on $X_{\zeta, i}^{\prime}$. If $e \geq 2$, then $Z_{\zeta,\left[\left(n_{0}-1\right) / 2\right]}^{\prime a}=Z_{\zeta}^{\prime a}$, and the claim follows. Assume that $e=1$. Then $m=1$. Let $X_{\zeta}^{\prime \prime}$ be the $\left(n_{0}+1\right)$-dimensional closed subvariety of $X_{\zeta}$ defined by

$$
u_{1}=0, \quad u_{4 j-1}=u_{4 j+2}, \quad u_{4 j}=u_{4 j+1} \quad \text { for } 1 \leq j \leq\left(n_{0}-2\right) / 2 .
$$

Then we have

$$
\operatorname{div}\left(z_{\zeta}-u_{n-2}-a\right)=\left[Z_{\zeta,\left[\left(n_{0}-1\right) / 2\right]}^{a}\right]+\left[Z_{\zeta}^{\prime a}\right]
$$

in $\mathrm{CH}_{n_{0}}\left(X_{\zeta}^{\prime \prime}\right)$, since we have

$$
\left(z-u_{n-2}\right)\left(z-u_{n-2}-1\right)=u_{n-2}\left(u_{n-3}+u_{n-2}+1\right)
$$

on $X_{\zeta}^{\prime \prime}$. Therefore, we obtain the claim.

Corollary 4.6 Assume that $n \geq 4$. Let $g$ be the automorphism of $X$ defined by

$$
\left(z,\left(y_{i}\right)_{1 \leq i \leq n-2}\right) \mapsto\left(z+\varepsilon_{1}\left(y_{n-2}+1\right), \sum_{i=1}^{n-3} y_{i}+\varepsilon_{1},\left(y_{i-1}+y_{n-2}+\varepsilon_{1}\right)_{2 \leq i \leq n-2}\right) .
$$

Then, $g^{*}$ acts on $H^{n-2}\left(X, \overline{\mathbb{Q}}_{\ell}\right)$ by -1 .

Proof Note that $g$ induces an automorphism of $X_{\zeta}$. The condition of $Z_{\zeta}^{0} \subset X_{\zeta}$ is equivalent to

$$
\begin{aligned}
& y_{4 i-3}+\zeta_{3}^{-1} y_{4 i-2}+\zeta_{3} y_{4 i-1}=0 \text { for } 1 \leq i \leq\left[n_{0} / 2\right], \\
& y_{4 i}+\zeta_{3} y_{4 i+1}+\zeta_{3}^{-1} y_{4 i+2}=0 \text { for } 1 \leq i \leq\left[\left(n_{0}-1\right) / 2\right], \\
& \zeta_{3}^{-1} y_{n-3}+\zeta_{3} y_{n-2}=0 \text { if } e \geq 2, \quad y_{n-2}=0 \text { if } e=1, \quad z_{\zeta}=0 .
\end{aligned}
$$

For $a \in \mathbb{F}_{2}$, the condition of $Z_{\zeta}^{\prime a} \subset X_{\zeta}$ is equivalent to

$$
\begin{aligned}
& \zeta_{3} y_{1}+\zeta_{3}^{-1} y_{2}+\sum_{j=3}^{n-2} y_{j}=0, \\
& y_{4 i-1}+\zeta_{3} y_{4 i}+\zeta_{3}^{-1} y_{4 i+1}=0, \quad y_{4 i}+\zeta_{3}^{-1} y_{4 i+1}+\zeta_{3} y_{4 i+2}=0 \\
& \quad \text { for } 1 \leq i \leq\left[\left(n_{0}-1\right) / 2\right], \\
& y_{n-3}=y_{n-2}+1 \text { if } e=1, \quad z_{\zeta}=a+\varepsilon_{1} y_{n-2} .
\end{aligned}
$$


Using the above, we can check that

$$
g^{-1}\left(Z_{\zeta}^{0}\right)= \begin{cases}Z_{\zeta}^{\prime 1} & \text { if } e=1 \\ Z_{\zeta}^{\prime 0} & \text { otherwise }\end{cases}
$$

Therefore, we obtain

$$
g^{*}\left(\operatorname{cl}\left(\left[Z_{\zeta}^{0}\right]\right)\right)=(-1)^{n_{0}+1}\left(\operatorname{cl}\left(\left[Z_{\zeta}^{\prime 0}\right]\right)\right)=-\operatorname{cl}\left(\left[Z_{\zeta}^{0}\right]\right)
$$

in $H^{n-2}\left(X_{\zeta}, \overline{\mathbb{Q}}_{\ell}\left(n_{0}\right)\right)$ using Lemma 4.3 and Proposition 4.5. Hence, the claim follows from Lemma 4.2 and Lemma 4.3.

\section{Explicit LLC and LJLC}

\subsection{Galois representations}

Let $X$ be the affine smooth variety over $k^{\text {ac }}$ defined by (2.13). We define an action of $Q \rtimes \mathbb{Z}$ on $X$ similarly to (3.18).

We choose an isomorphism $\iota: \overline{\mathbb{Q}}_{\ell} \simeq \mathbb{C}$. Let $q^{1 / 2} \in \overline{\mathbb{Q}}_{\ell}$ be the 2-nd root of $q$ such that $\iota\left(q^{1 / 2}\right)>0$. For a rational number $r \in 2^{-1} \mathbb{Z}$, let $\overline{\mathbb{Q}}_{\ell}(r)$ be the unramified representation of $\mathrm{Gal}\left(k^{\mathrm{ac}} / k\right)$ of degree 1 , on which the geometric Frobenius $\mathrm{Frob}_{q}$ acts as scalar multiplication by $q^{-r}$. We simply write $Q$ for the subgroup $Q \times\{0\} \subset Q \rtimes \mathbb{Z}$. We consider the morphisms

$$
\begin{aligned}
& \Phi: \mathbb{A}_{k^{\mathrm{ac}}}^{n-1} \rightarrow \mathbb{A}_{k^{\mathrm{ac}}}^{1} ;\left(y,\left(y_{i}\right)_{1 \leq i \leq n-2}\right) \mapsto y^{p^{e}+1}-\frac{1}{n^{\prime}} \sum_{1 \leq i \leq j \leq n-2} y_{i} y_{j}, \\
& h_{m}: \mathbb{A}_{k^{\mathrm{ac}}}^{1} \rightarrow \mathbb{A}_{k^{\mathrm{ac}}}^{1} ; z \mapsto z^{p^{m}}-z .
\end{aligned}
$$

Then we have a cartesian diagram

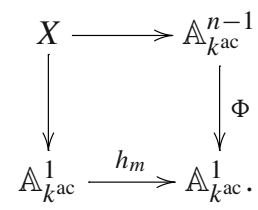

Using the proper base change theorem for the above cartesian diagram, we have a decomposition

$$
H_{\mathrm{c}}^{n-1}\left(X, \overline{\mathbb{Q}}_{\ell}\right) \simeq \underset{\psi \in \mathbb{F}_{p^{m}}^{\vee} \backslash\{1\}}{\bigoplus} H_{\mathrm{c}}^{n-1}\left(\mathbb{A}_{k^{\mathrm{ac}}}^{n-1}, \mathcal{L}_{\psi}(\Phi)\right),
$$


since $h_{m *} \overline{\mathbb{Q}}_{\ell} \simeq \bigoplus_{\psi \in \mathbb{F}_{p^{m}}^{\vee}} \mathcal{L}_{\psi}$ and $H_{\mathrm{c}}^{n-1}\left(\mathbb{A}_{k^{\mathrm{ac}}}^{n-1}, \overline{\mathbb{Q}}_{\ell}\right)=0$. The decomposition (5.1) is stable under the action of $Q \rtimes \mathbb{Z}$, since $\mathbb{F}_{p^{m}} \simeq\left\{g(1,0, c) \mid c \in \mathbb{F}_{p^{m}}\right\}$ in the center of $Q \rtimes \mathbb{Z}$ acts on each direct summand $H_{\mathrm{c}}^{n-1}\left(\mathbb{A}_{k^{\mathrm{ac}}}^{n-1}, \mathcal{L}_{\psi}(\Phi)\right)$ in $(5.1)$ by $\psi$. We put

$$
\tau_{\psi, n}=H_{\mathrm{c}}^{n-1}\left(\mathbb{A}_{k^{\mathrm{ac}}}^{n-1}, \mathcal{L}_{\psi}(\Phi)\right)\left(\frac{n-1}{2}\right)
$$

as a $Q \rtimes \mathbb{Z}$-representation for each $\psi \in \mathbb{F}_{p^{m}}^{\vee} \backslash\{1\}$. We write $\tau_{r, \psi}^{0}$ for the inflation of $\tau_{\psi, n}$ by $\Theta_{r}$ in (3.19).

\subsection{Correspondence}

Definition 5.1 We say that an irreducible supercuspidal representation of $G L_{n}(K)$ is simple supercuspidal if its exponential Swan conductor is one.

Remark 5.2 Definition 5.1 is compatible with [17, Definition 1.1] by [17, Proposition 1.3]. The word "simple supercuspidal" comes from [7]. Our "simple supercuspidal" representations are called "epipelagic" in [2] after [20].

We define $\psi_{0} \in \mathbb{F}_{p}^{\vee}$ by $\iota\left(\psi_{0}(1)\right)=\exp (2 \pi \sqrt{-1} / p)$. We put $\psi_{0}^{\prime}=\psi_{0} \circ \operatorname{Tr}_{\mathbb{F}_{p} m} / \mathbb{F}_{p}$. We take an additive character $\psi_{K}: K \rightarrow \overline{\mathbb{Q}}_{\ell}^{\times}$such that $\psi_{K}(x)=\psi_{0}^{\prime}(\bar{x})$ for $x \in \mathcal{O}_{K}$. In the following, for each triple $(\zeta, \chi, c) \in \mu_{q-1}(K) \times\left(k^{\times}\right)^{\vee} \times \overline{\mathbb{Q}}_{\ell}^{\times}$, we define a $G L_{n}(K)$-representation $\pi_{\zeta, \chi, c}$, a $D^{\times}$-representation $\rho_{\zeta, \chi, c}$ and a $W_{K}$-representation $\tau_{\zeta, \chi, c}$.

We use notations in Sect. 2.1, replacing $r \in \mu_{q-1}(K)$ with $\zeta \in \mu_{q-1}(K)$. We have the $K$-algebra embeddings

$$
L_{\zeta} \rightarrow M_{n}(K) ; \varphi_{\zeta} \mapsto \varphi_{M, \zeta}, \quad L_{\zeta} \rightarrow D ; \varphi_{\zeta} \mapsto \varphi_{D, \zeta}
$$

Set $\varphi_{\zeta, n}=n^{\prime} \varphi_{\zeta}$. Let $\Lambda_{\zeta, \chi, c}: L_{\zeta}^{\times} U_{\Im}^{1} \rightarrow \overline{\mathbb{Q}}_{\ell}^{\times}$be the character defined by

$$
\begin{aligned}
\Lambda_{\zeta, \chi, c}\left(\varphi_{\zeta}\right) & =(-1)^{n-1} c, \quad \Lambda_{\zeta, \chi, c}(x)=\chi(\bar{x}) \quad \text { for } x \in \mathcal{O}_{K}^{\times}, \\
\Lambda_{\zeta, \chi, c}(x) & =\left(\psi_{K} \circ \operatorname{tr}\right)\left(\varphi_{\zeta, n}^{-1}(x-1)\right) \text { for } x \in U_{\Im}^{1} .
\end{aligned}
$$

We put

$$
\pi_{\zeta, \chi, c}=\mathrm{c}-\operatorname{Ind}_{L_{\zeta}^{\times} U_{\mathfrak{I}}^{1}}^{G L_{n}(K)} \Lambda_{\zeta, \chi, c}
$$

Then, $\pi_{\zeta, \chi, c}$ is a simple supercuspidal representation of $G L_{n}(K)$, and every simple supercuspidal representation is isomorphic to $\pi_{\zeta, \chi, c}$ for a uniquely determined $(\zeta, \chi, c) \in \mu_{q-1}(K) \times\left(k^{\times}\right)^{\vee} \times \overline{\mathbb{Q}}_{\ell}^{\times}(c f .[2,2.1,2.2])$.

Let $\theta_{\zeta, \chi, c}: L_{\zeta}^{\times} U_{D}^{1} \rightarrow \overline{\mathbb{Q}}_{\ell}^{\times}$be the character defined by

$$
\theta_{\zeta, \chi, c}\left(\varphi_{\zeta}\right)=c, \quad \theta_{\zeta, \chi, c}(x)=\chi(\bar{x}) \text { for } x \in \mathcal{O}_{K}^{\times},
$$




$$
\theta_{\zeta, \chi, c}(d)=\left(\psi_{K} \circ \operatorname{Trd}_{D / K}\right)\left(\varphi_{\zeta, n}^{-1}(d-1)\right) \text { for } d \in U_{D}^{1}
$$

We put

$$
\rho_{\zeta, \chi, c}=\operatorname{Ind}_{L_{\zeta}^{\times} U_{D}^{1}}^{D^{\times}} \theta_{\zeta, \chi, c} .
$$

The isomorphism class of this representation does not depend on the choice of the embedding $L_{\zeta} \hookrightarrow D$.

Recall that $\varphi_{\zeta}^{\prime}=\varphi_{\zeta}^{p^{e}}$ and $E_{\zeta}=K\left(\varphi_{\zeta}^{\prime}\right)$. Let $\phi_{c}: W_{E_{\zeta}} \rightarrow \overline{\mathbb{Q}}_{\ell}^{\times}$be the character defined by $\phi_{c}(\sigma)=c^{n_{\sigma}}$. Let Frob ${ }_{p}: k^{\times} \rightarrow k^{\times}$be the map defined by $x \mapsto x^{p^{-1}}$ for $x \in k^{\times}$. We consider the composite

$$
v_{\zeta}: W_{E_{\zeta}}^{\mathrm{ab}} \stackrel{\operatorname{Art}_{E_{\zeta}}^{-1}}{\longrightarrow} E_{\zeta}^{\times} \rightarrow \mathcal{O}_{E_{\zeta}}^{\times} \stackrel{\mathrm{can}^{\prime}}{\longrightarrow} k^{\times} \stackrel{\operatorname{Frob}_{p}^{e}}{\longrightarrow} k^{\times},
$$

where the second homomorphism is given by $E_{\zeta}^{\times} \rightarrow \mathcal{O}_{E_{\zeta}}^{\times} ; x \mapsto x \varphi_{\zeta}^{\prime-v_{E_{\zeta}}(x)}$. We simply write $\tau_{\zeta}^{0}$ for $\tau_{\zeta, \psi_{0}^{\prime}}^{0}$. We set

$$
\tau_{\zeta, \chi, c}^{0}=\tau_{\zeta}^{0} \otimes\left(\chi \circ v_{\zeta}\right) \otimes \phi_{c}, \quad \tau_{\zeta, \chi, c}=\operatorname{Ind}_{W_{E_{\zeta}}}^{W_{K}} \tau_{\zeta, \chi, c}^{0} .
$$

We see that $\tau_{\zeta, \chi, c}^{0}$ is primitive by [2, 3.2 Proposition] and [13].

The following theorem follows from [13] and [17].

Theorem 5.3 Let LL and JL denote the local Langlands correspondence and the local Jacquet-Langlands correspondence for $G L_{n}(K)$ respectively. For $\zeta \in \mu_{q-1}(K), \chi \in$ $\left(k^{\times}\right)^{\vee}$ and $c \in \overline{\mathbb{Q}}_{\ell}^{\times}$, we have $\operatorname{LL}\left(\pi_{\zeta, \chi, c}\right)=\tau_{\zeta, \chi, c}$ and $\operatorname{JL}\left(\rho_{\zeta, \chi, c}\right)=\pi_{\zeta, \chi, c}$.

Definition 5.4 We say that a smooth irreducible representation of $G L_{n}(K)$ is essentially simple supercuspidal if it is a character twist of a simple supercuspidal representation.

Let $\omega: K^{\times} \rightarrow \overline{\mathbb{Q}}_{\ell}^{\times}$be a smooth character. We put

$$
\begin{aligned}
\pi_{\zeta, \chi, c, \omega} & =\pi_{\zeta, \chi, c} \otimes(\omega \circ \mathrm{det}), \quad \rho_{\zeta, \chi, c, \omega}=\rho_{\zeta, \chi, c} \otimes\left(\omega \circ \operatorname{Nrd}_{D / K}\right), \\
\tau_{\zeta, \chi, c, \omega} & =\tau_{\zeta, \chi, c} \otimes\left(\omega \circ \mathrm{Art}_{K}^{-1}\right),
\end{aligned}
$$

and

$$
\begin{aligned}
\Lambda_{\zeta, \chi, c, \omega} & =\Lambda_{\zeta, \chi, c} \otimes\left(\left.\omega \circ \operatorname{det}\right|_{L_{\zeta}^{\times} U_{\mathfrak{J}}^{1}}\right), \quad \theta_{\zeta, \chi, c, \omega}=\theta_{\zeta, \chi, c} \otimes\left(\left.\omega \circ \operatorname{Nrd}_{D / K}\right|_{L_{\zeta}^{\times} U_{D}^{1}}\right), \\
\tau_{\zeta, \chi, c, \omega}^{0} & =\tau_{\zeta, \chi, c}^{0} \otimes\left(\omega \circ \operatorname{Nr}_{E_{\zeta} / K} \circ \operatorname{Art}_{E_{\zeta}}^{-1}\right) .
\end{aligned}
$$

Then we have

$$
\pi_{\zeta, \chi, c, \omega}=\mathrm{c}-\operatorname{Ind}_{L_{\zeta}^{\times} U_{\mathfrak{I}}^{1}}^{G L_{n}(K)} \Lambda_{\zeta, \chi, c, \omega},
$$




$$
\rho_{\zeta, \chi, c, \omega}=\operatorname{Ind}_{L_{\zeta}^{\times} U_{D}^{1}}^{D^{\times}} \theta_{\zeta, \chi, c, \omega}, \quad \tau_{\zeta, \chi, c, \omega}=\operatorname{Ind}_{W_{E_{\zeta}} W_{K}} \tau_{\zeta, \chi, c, \omega}^{0} .
$$

Corollary 5.5 We have $\operatorname{LL}\left(\pi_{\zeta, \chi, c, \omega}\right)=\tau_{\zeta, \chi, c, \omega}$ and $\operatorname{JL}\left(\rho_{\zeta, \chi, c, \omega}\right)=\pi_{\zeta, \chi, c, \omega}$.

Proof This follows from Theorem 5.3, because LL and JL are compatible with character twists.

\section{Geometric realization}

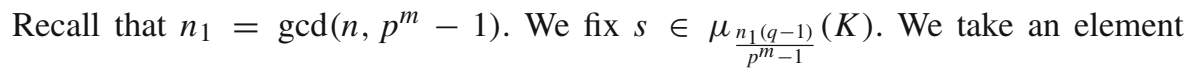
$r \in \mu_{q-1}(K)$ such that $r^{\frac{p^{m}-1}{n_{1}}}=s$. We put

$$
H_{\mathfrak{X}_{r}}=H_{\mathrm{c}}^{n-1}\left(\overline{\mathfrak{X}}_{r}, \overline{\mathbb{Q}}_{\ell}\right)\left(\frac{n-1}{2}\right)
$$

as $H_{r}$-representations.

Lemma 6.1 The isomorphism class of c-Ind $H_{r}^{G} H_{\mathfrak{X}_{r}}$ depends only on s.

Proof Assume that $r, r^{\prime} \in \mu_{q-1}(K)$ satisfy

$$
r^{\frac{p^{m}-1}{n_{1}}}=r^{\prime \frac{p^{m}-1}{n_{1}}}=s .
$$

Then we have $L_{r}=L_{r^{\prime}}$. Hence, there is $(g, d) \in\left(G L_{n}(K) \times D^{\times}\right)^{0}$ such that $\xi_{r}(g, d)=\xi_{r^{\prime}}$ by Lemma 1.6. Then we have $\mathfrak{X}_{r}(g, d)=\mathfrak{X}_{r^{\prime}}$. Threfore we obtain the calim.

We put

$$
\Pi_{s}=\mathrm{c}-\operatorname{Ind}_{H_{r}}^{G} H_{\mathfrak{X}_{r}} .
$$

For simplicity, we write $G_{1}$ and $G_{2}$ for $G L_{n}(K)$ and $D^{\times} \times W_{K}$ respectively, and consider them as subgroups of $G$. We put

$$
H=\left\{g \in U_{\mathfrak{I}}^{1} \mid \operatorname{det}(g)=1\right\} .
$$

We have $H=H_{r} \cap G_{1}$ by Proposition 3.3. Let $\bar{H}_{r}$ be the image of $H_{r}$ in $G / G_{1} \simeq G_{2}$.

Let $a \in \mu_{q-1}(K)$. We define a character $\Lambda_{r}^{a}: U_{\mathfrak{I}}^{1} \rightarrow \overline{\mathbb{Q}}_{\ell}^{\times}$by

$$
\Lambda_{r}^{a}(x)=\left(\psi_{K} \circ \operatorname{tr}\right)\left(\left(a \varphi_{r, n}\right)^{-1}(x-1)\right) \quad \text { for } x \in U_{\mathfrak{I}}^{1} .
$$

Let $\pi$ be a smooth irreducible representation of $G L_{n}(K)$. 
Lemma 6.2 If $\pi$ is not essentially simple supercuspidal, then we have $\operatorname{Hom}_{H}\left(\Lambda_{r}^{a}, \pi\right)=$ 0 . Further, we have

$$
\operatorname{dim} \operatorname{Hom}_{H}\left(\Lambda_{r}^{a}, \pi_{\zeta, \chi, c, \omega}\right)= \begin{cases}1 & \text { if } a^{n} r=\zeta \\ 0 & \text { otherwise }\end{cases}
$$

Proof We assume that $\operatorname{Hom}_{H}\left(\Lambda_{r}^{a}, \pi\right) \neq 0$, and show that $\pi$ is essentially simple supercuspidal. Let $\omega_{\pi}$ be the central character of $\pi$. Then $\omega_{\pi}$ is trivial on $K^{\times} \cap H$ by $\operatorname{Hom}_{H}\left(\Lambda_{r}^{a}, \pi\right) \neq 0$. Hence, we may assume that $\omega_{\pi}$ is trivial on $K^{\times} \cap U_{\mathfrak{I}}^{1}$, changing $\pi$ by a character twist. Then, there is a character $\Lambda_{r, \omega_{\pi}}^{a}: K^{\times} U_{\mathfrak{I}}^{1} \rightarrow \overline{\mathbb{Q}}_{\ell}^{\times}$such that

$$
\left.\Lambda_{r, \omega_{\pi}}^{a}\right|_{U_{\mathfrak{I}}^{1}}=\Lambda_{r}^{a},\left.\quad \Lambda_{r, \omega_{\pi}}^{a}\right|_{K^{\times}}=\omega_{\pi}
$$

Then we have

$$
\operatorname{Hom}_{H}\left(\Lambda_{r}^{a}, \pi\right) \simeq \operatorname{Hom}_{K^{\times} H}\left(\Lambda_{r, \omega_{\pi}}^{a}, \pi\right) \simeq \operatorname{Hom}_{K^{\times} U_{\Im}^{1}}\left(\operatorname{Ind}_{K^{\times} H}^{K^{\times} U_{\Im}^{1}}\left(\left.\Lambda_{r, \omega_{\pi}}^{a}\right|_{K^{\times} H}\right), \pi\right)
$$

by Frobenius reciprocity, since $K^{\times} U_{\mathfrak{I}}^{1} /\left(K^{\times} H\right)$ is compact. We have the natural isomorphism

$$
K^{\times} U_{\mathfrak{I}}^{1} /\left(K^{\times} H\right) \stackrel{\operatorname{det}}{\longrightarrow}\left(K^{\times}\right)^{n} U_{K}^{1} /\left(K^{\times}\right)^{n} \simeq U_{K}^{1} /\left(U_{K}^{1}\right)^{n} .
$$

For a smooth character $\phi$ of $U_{K}^{1} /\left(U_{K}^{1}\right)^{n}$, let $\phi^{\prime}$ denote the character of $K^{\times} U_{\mathfrak{I}}^{1}$ obtained by $\phi$ and the isomorphism (6.2). We have a natural isomorphism

$$
\operatorname{Ind}_{K^{\times} H}^{K^{\times} U_{\Im}^{1}}\left(\left.\Lambda_{r, \omega_{\pi}}^{a}\right|_{K^{\times} H}\right) \simeq \bigoplus_{\phi \in\left(U_{K}^{1} /\left(U_{K}^{1}\right)^{n}\right)^{\vee}} \Lambda_{r, \omega_{\pi}}^{a} \otimes \phi^{\prime} .
$$

Let $\phi$ be a smooth character of $U_{K}^{1} /\left(U_{K}^{1}\right)^{n}$, and regard it as a character of $U_{K}^{1}$. We extend $\phi$ to a character $\tilde{\phi}$ of $K^{\times}$such that $\tilde{\phi}(\varpi)=1$ and $\tilde{\phi}$ is trivial on $\mu_{q-1}(K)$. We have

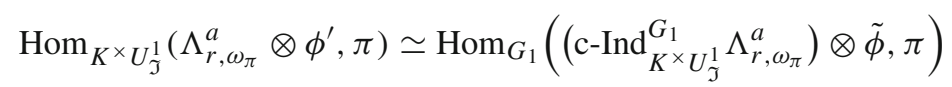

by Frobenius reciprocity. We take $\chi^{\prime} \in\left(k^{\times}\right)^{\vee}$ such that $\chi^{\prime}(\bar{x})=\omega_{\pi}(x)$ for $x \in$ $\mu_{q-1}(K)$. For $c^{\prime} \in \overline{\mathbb{Q}}_{\ell}^{\times}$, we define the character $\Lambda_{r, \chi^{\prime}, c^{\prime}}^{a}: L_{r}^{\times} U_{\mathfrak{I}}^{1} \rightarrow \overline{\mathbb{Q}}_{\ell}^{\times}$by

$$
\left.\Lambda_{r, \chi^{\prime}, c^{\prime}}^{a}\right|_{U_{\mathfrak{J}}}=\Lambda_{r}^{a}, \quad \Lambda_{r, \chi^{\prime}, c^{\prime}}^{a}\left(\varphi_{M, r}\right)=c^{\prime}, \quad \Lambda_{r, \chi^{\prime}, c^{\prime}}^{a}(x)=\chi^{\prime}(\bar{x}) \quad \text { for } x \in \mu_{q-1}(K) .
$$

We put

$$
\pi_{r, \chi^{\prime}, c^{\prime}}^{a}=\mathrm{c}-\operatorname{Ind}_{L_{r}^{\times} U_{\mathfrak{I}}^{1}}^{G_{1}} \Lambda_{r, \chi^{\prime}, c^{\prime}}^{a} .
$$


Then we have

$$
\mathrm{c}-\operatorname{Ind}_{K^{\times} U_{\mathfrak{I}}^{1}}^{G_{1}} \Lambda_{r, \omega_{\pi}}^{a} \simeq \bigoplus_{c^{\prime} \in \overline{\mathbb{Q}}_{\ell}^{\times}} \pi_{r, \chi^{\prime}, c^{\prime}}^{a}
$$

Note that

$$
\pi_{r, \chi^{\prime}, c^{\prime}}^{a} \simeq \pi_{a^{n} r, \chi^{\prime}, \chi^{\prime}(a) c^{\prime}}
$$

by the constructions. Then we see that $\pi$ is simple supercuspidal by (6.1), (6.3), (6.4), (6.5), (6.6) and the assumption $\operatorname{Hom}_{H}\left(\Lambda_{r}^{a}, \pi\right) \neq 0$.

Let $\chi^{\prime} \in\left(k^{\times}\right)^{\vee}$. We use the same notations as above for such $\chi^{\prime}$. For an irreducible supercuspidal representation $\pi$ of $G_{1}$, we write a $(\pi)$ for its Artin conductor exponent as in $[2,1.2]$. We have a $\left(\pi_{r, \chi^{\prime}, c^{\prime}}^{a}\right)=n+1$ by (6.6). Hence, if $\phi \neq 1$, we have

$$
\mathrm{a}\left(\pi_{r, \chi^{\prime}, c^{\prime}}^{a} \otimes \tilde{\phi}\right)=n \mathrm{a}(\tilde{\phi}) \geq 2 n
$$

by a $(\tilde{\phi}) \geq 2$ and $[3,6.5$ Theorem (ii)]. Therefore, we obtain

$$
\operatorname{dim} \operatorname{Hom}_{G_{1}}\left(\pi_{r, \chi^{\prime}, c^{\prime}}^{a} \otimes \tilde{\phi}, \pi_{\zeta, \chi, c}\right)= \begin{cases}1 & \text { if } \phi=1, a^{n} r=\zeta \text { and } \chi^{\prime}(a) c^{\prime}=c \\ 0 & \text { otherwise }\end{cases}
$$

by $(6.6)$ and $[2,2.2]$. To show the second claim, we may assume that $\omega=1$. Hence, we obtain the second claim by the above discussion, using that $\omega_{\pi_{\zeta, \chi, c}}$ is trivial on $U_{K}^{1}$.

Proposition 6.3 (1) If $\pi$ is not essentially simple supercuspidal, we have $\operatorname{Hom}_{H}\left(H_{\mathfrak{X}_{r}}, \pi\right)=0$. Further, we have

$$
\operatorname{dim} \operatorname{Hom}_{H}\left(H_{\mathfrak{X}_{r}}, \pi_{\zeta, \chi, c, \omega}\right)= \begin{cases}p^{e} n_{1} & \text { if } \zeta^{\frac{p^{m}-1}{n_{1}}}=s \\ 0 & \text { otherwise }\end{cases}
$$

(2) We have $L_{r}^{\times} U_{D}^{1} \times W_{E_{r}} \subset \bar{H}_{r}$ and an injective homomorphism

$$
\theta_{r, \chi, c, \omega} \otimes \tau_{r, \chi, c, \omega}^{0} \hookrightarrow \operatorname{Hom}_{H}\left(H_{\mathfrak{X}_{r}}, \pi_{r, \chi, c, \omega}\right)
$$

as $L_{r}^{\times} U_{D}^{1} \times W_{E_{r}}$-representations.

Proof By (5.1), we have a decomposition

$$
H_{\mathfrak{X}_{r}} \simeq \bigoplus_{\psi \in \mathbb{F}_{p^{m}}^{\vee} \backslash\{1\}} \tau_{\psi, n}
$$

as representations of $Q \rtimes \mathbb{Z}$. By Proposition 3.3 and (6.7), we have

$$
H_{\mathfrak{X}_{r}} \simeq \bigoplus_{a \in \mu_{p^{m}-1}(K)}\left(\Lambda_{r}^{-a}\right)^{\oplus p^{e}}
$$


as $H$-representations. By (6.8), we have

$$
\operatorname{Hom}_{H}\left(H_{\mathfrak{X}_{r}}, \pi_{\zeta, \chi, c, \omega}\right) \simeq \bigoplus_{a \in \mu_{p^{m}-1}(K),(-a)^{n} r=\zeta} \operatorname{Hom}_{H}\left(\Lambda_{r}^{-a}, \pi_{\zeta, \chi, c, \omega}\right)^{\oplus p^{e}}
$$

The cardinality of

$$
\left\{a \in \mu_{p^{m}-1}(K) \mid(-a)^{n} r=\zeta\right\}
$$

equals $n_{1}$ if $\zeta^{\frac{p^{m}-1}{n_{1}}}=s$ and zero otherwise. Hence the first claim follows from Lemma 6.2 .

We prove the second claim. We consider the element

$$
\left(\varphi_{D, r}, 1\right) \in L_{r}^{\times} U_{D}^{1} \times W_{E_{r}} \subset G_{2}
$$

and its lifting $\mathbf{g}_{r} \in G$ in (3.1) with respect to $G \rightarrow G_{2}$. We have $\mathbf{g}_{r} \in H_{r}$ by Proposition 3.2 (1). The element $\left(\varphi_{D, r}, 1\right)$ acts on $\theta_{r, \chi, c, \omega} \otimes \tau_{r, \chi, c, \omega}^{0}$ as scalar multiplication by $c \omega\left((-1)^{n-1} \varpi_{r}\right)$, because $\operatorname{Nrd}_{D / K}\left(\varphi_{D, r}\right)=(-1)^{n-1} \varpi_{r}$. By Proposition 3.2 (2), Corollary 4.6 and [12, Proposition 4.2.3], the element $\mathbf{g}_{r}$ acts on $\operatorname{Hom}_{H}\left(H_{\mathfrak{X}_{r}}, \pi_{r, \chi, c, \omega}\right)$ as scalar multiplication by $c \omega\left((-1)^{n-1} \varpi_{r}\right)$.

Let $z d \in \mathcal{O}_{K}^{\times} U_{D}^{1}$ with $z \in \mu_{q-1}(K)$ and $d \in U_{D}^{1}$. Let $g=\left(a_{i, j}\right)_{1 \leq i, j \leq n} \in U_{\mathfrak{I}}^{1}$ be the element defined by $a_{1,1}=\operatorname{Nrd}_{D / K}(d), a_{i, i}=1$ for $2 \leq i \leq n$ and $a_{i, j}=0$ if $i \neq j$. We have $\operatorname{det}(g)=\operatorname{Nrd}_{D / K}(d)$ and $(z g, z d, 1) \in H_{r}$. The element $(z d, 1) \in$ $L_{r}^{\times} U_{D}^{1} \times W_{E_{r}}$ acts on $\theta_{r, \chi, c, \omega} \otimes \tau_{r, \chi, c, \omega}^{0}$ as scalar multiplication by

$$
\chi(\bar{z}) \theta_{r, \chi, c}(d) \omega\left(\operatorname{Nrd}_{D / K}(z d)\right)
$$

We have the subspace

$$
\operatorname{Hom}_{H}\left(\tau_{\psi_{0}^{\prime-1}, n}, \pi_{r, \chi, c, \omega}\right) \subset \operatorname{Hom}_{H}\left(H_{\mathfrak{X}_{r}}, \pi_{r, \chi, c, \omega}\right)
$$

by the decomposition (6.7). By Remark 1.4, Proposition 3.3 and [12, Propositions 4.2.1 and 4.5.1], the element $(z g, z d, 1)$ acts on the subspace in (6.9) as scalar multiplication by

$$
\chi(\bar{z}) \theta_{r, \chi, c}(d) \omega(\operatorname{det}(z g))
$$

Let $\sigma \in W_{E_{r}}$ such that $n_{\sigma}=1$. We take $\mathbf{g}_{\sigma}$ as in (3.15). By Proposition 3.4, the element $\mathbf{g}_{\sigma}$ acts on the subspace (6.9) by

$$
\chi\left(\bar{b}_{\sigma}\right) \tau_{r, \psi_{0}^{\prime}}^{0}(\sigma) \omega\left(\operatorname{det}\left(g_{\sigma}\right)\right)
$$


On the other hand, the element $\left(\varphi_{D, r}^{-1}, \sigma\right) \in L_{r}^{\times} U_{D}^{1} \times W_{E_{r}}$ acts on $\theta_{r, \chi, c, \omega} \otimes \tau_{r, \chi, c, \omega}^{0}$ by

$$
\left(\chi \circ v_{r}\right)(\sigma) \tau_{r, \psi_{0}^{\prime}}^{0}(\sigma) \omega\left(\mathrm{Nr}_{E_{r} / K}\left(u_{\sigma}\right)\right)
$$

Hence, the required assertion follows from $v_{r}(\sigma)=\bar{b}_{\sigma}$ and $\operatorname{Nr}_{E_{r} / K}\left(u_{\sigma}\right)=\operatorname{det}\left(g_{\sigma}\right)$.

Proposition 6.4 If $\pi$ is not essentially simple supercuspidal, then we have $\operatorname{Hom}_{G L_{n}(K)}\left(\Pi_{s}, \pi\right)=0$. Further, we have

$$
\operatorname{Hom}_{G L_{n}(K)}\left(\Pi_{s}, \pi_{\zeta, \chi, c, \omega}\right) \simeq \begin{cases}\rho_{\zeta, \chi, c, \omega} \otimes \tau_{\zeta, \chi, c, \omega} & \text { if } \zeta^{\frac{p^{m}-1}{n_{1}}}=s \\ 0 & \text { otherwise }\end{cases}
$$

as $D^{\times} \times W_{K}$-representations.

Proof For $g \in H_{r} \backslash G / G_{1}$, we choose an element $\tilde{g} \in G_{2}$ whose image in $\bar{H}_{r} \backslash G_{2}$ equals $g$ under the natural isomorphism $H_{r} \backslash G / G_{1} \simeq \bar{H}_{r} \backslash G_{2}$. We put $H^{\tilde{g}}=\tilde{g}^{-1} H \tilde{g}$. Let $H_{\mathfrak{X}_{r}}^{\tilde{g}}$ denote the representation of $H^{\tilde{g}}$ which is the conjugate of $H_{\mathfrak{X}_{r}}$ by $\tilde{g}$. Then, we have

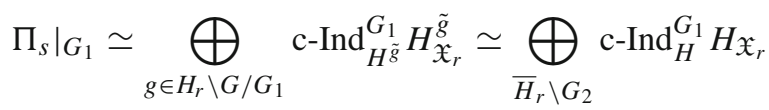

as $G_{1}$-representations by Mackey's decomposition theorem, since we have $H^{\tilde{g}}=H$ and $H_{\mathfrak{X}_{r}} \simeq H_{\mathfrak{X}_{r}}^{\tilde{g}}$ as $H$-representations. By (6.10) and Frobenius reciprocity, we acquire

$$
\operatorname{Hom}_{G_{1}}\left(\Pi_{s}, \pi_{\zeta, \chi, c, \omega}\right) \simeq \bigoplus_{\bar{H}_{r} \backslash G_{2}} \operatorname{Hom}_{H}\left(H_{\mathfrak{X}_{r}}, \pi_{\zeta, \chi, c, \omega}\right)
$$

If $\zeta^{\frac{p^{m}-1}{n_{1}}} \neq s$, the required assertion follows from (6.11) and Proposition 6.3 (1). Now, assume that $\zeta^{\frac{p^{m}-1}{n_{1}}}=s$. Without loss of generality, we may assume that $\zeta$ equals $r$ by Lemma 6.1. By Proposition 6.3 and Frobenius reciprocity, we obtain a non-zero map

$$
\operatorname{Ind}_{L_{r}^{\times} U_{D}^{1} \times W_{E_{r}}}^{\bar{H}_{r}}\left(\theta_{r, \chi, c, \omega} \otimes \tau_{r, \chi, c, \omega}^{0}\right) \rightarrow \operatorname{Hom}_{H}\left(H_{\mathfrak{X}_{r}}, \pi_{r, \chi, c, \omega}\right) .
$$

By applying Ind $\bar{H}_{r}$ to the map (6.12), we acquire a non-zero map

$$
\rho_{r, \chi, c, \omega} \otimes \tau_{r, \chi, c, \omega} \rightarrow \operatorname{Ind}_{H_{r}}^{G_{2}} \operatorname{Hom}_{H}\left(H_{\mathfrak{X}_{r}}, \pi_{r, \chi, c, \omega}\right) .
$$

We have $\operatorname{dim} \rho_{r, \chi, c, \omega}=\left(q^{n}-1\right) /(q-1)$ and $\operatorname{dim} \tau_{r, \chi, c, \omega}=n$. Moreover, we have

$$
\left[G_{2}: \bar{H}_{r}\right]=\left[F_{r}: K\right]\left[D^{\times}: L_{r}^{\times} U_{D}^{1}\right]=\frac{n^{\prime}\left(q^{n}-1\right)}{n_{1}(q-1)}
$$


by the exact sequence

$$
1 \rightarrow L_{r}^{\times} U_{D}^{1} \rightarrow \bar{H}_{r} \rightarrow W_{F_{r}} \rightarrow 1
$$

Hence, the both sides of (6.13) are $n\left(q^{n}-1\right) /(q-1)$-dimensional by Proposition 6.3 (1). Since $\rho_{r, \chi, c, \omega} \otimes \tau_{r, \chi, c, \omega}$ is an irreducible representation of $G_{2}$, we know that (6.13) is an isomorphism as $G_{2}$-representations. On the other hand, we have a non-zero map

$$
\operatorname{Ind} \bar{H}_{r}^{G_{2}} \operatorname{Hom}_{H}\left(H_{\mathfrak{X}_{r}}, \pi_{r, \chi, c, \omega}\right) \rightarrow \operatorname{Hom}_{G_{1}}\left(\Pi_{s}, \pi_{r, \chi, c, \omega}\right)
$$

induced by a surjective homomorphism $\left.\Pi_{s}\right|_{H_{r}} \rightarrow H_{\mathfrak{X}_{r}}$ of $H_{r}$-representations and Frobenius reciprocity. Then (6.14) is an isomorphism, since the left hand side is an irreducible representation of $G_{2}$ and the both sides have the same dimension by (6.11). Hence, the required assertion follows from the isomorphisms (6.13) and (6.14).

Theorem 6.5 Let $\mathrm{LJ}$ be the inverse of JL in Proposition 5.3. We put

$$
\Pi=\bigoplus_{s \in \mu_{\frac{n_{1}(q-1)}{p^{m}-1}}(K)} \Pi_{s}
$$

Let $\pi$ be a smooth irreducible representation of $G L_{n}(K)$. Then, we have

$$
\operatorname{Hom}_{G L_{n}(K)}(\Pi, \pi) \simeq \begin{cases}\operatorname{LJ}(\pi) \otimes \operatorname{LL}(\pi) & \text { if } \pi \text { is essentially simple supercuspidal, } \\ 0 & \text { otherwise }\end{cases}
$$

as $D^{\times} \times W_{K}$-representations.

Proof This follows from Proposition 5.3 and Lemma 6.4, because every essentially simple supercuspidal representation is isomorphic to $\pi_{\zeta, \chi, c, \omega}$ for some $\zeta \in \mu_{q-1}(K)$, $\chi \in\left(k^{\times}\right)^{\vee}, c \in \overline{\mathbb{Q}}_{\ell}^{\times}$and a smooth character $\omega: K^{\times} \rightarrow \overline{\mathbb{Q}}_{\ell}^{\times}$.

Acknowledgements The authors would like to thank a referee for helpful comments and suggestions. This work was supported by JSPS KAKENHI Grant Numbers 26707003, 15K17506, 18H01109, 20K03529.

Open Access This article is licensed under a Creative Commons Attribution 4.0 International License, which permits use, sharing, adaptation, distribution and reproduction in any medium or format, as long as you give appropriate credit to the original author(s) and the source, provide a link to the Creative Commons licence, and indicate if changes were made. The images or other third party material in this article are included in the article's Creative Commons licence, unless indicated otherwise in a credit line to the material. If material is not included in the article's Creative Commons licence and your intended use is not permitted by statutory regulation or exceeds the permitted use, you will need to obtain permission directly from the copyright holder. To view a copy of this licence, visit http://creativecommons.org/licenses/by/4.0/.

\section{References}

1. Bushnell, C.J., Henniart, G.: The essentially tame local Langlands correspondence, I. J. Am. Math. Soc. 18(3), 685-710 (2005) 


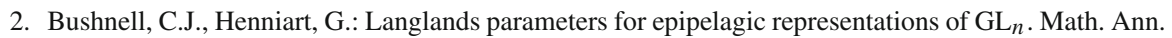
358(1-2), 433-463 (2014)

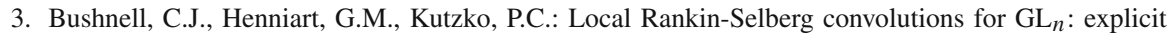
conductor formula. J. Am. Math. Soc. 11(3), 703-730 (1998)

4. Boyer, P.: Mauvaise réduction des variétés de Drinfeld et correspondance de Langlands locale. Invent. Math. 138(3), 573-629 (1999)

5. Boyarchenko, M., Weinstein, J.: Maximal varieties and the local Langlands correspondence for $G L(n)$. J. Am. Math. Soc. 29(1), 177-236 (2016)

6. Deligne, P.: Cohomologie étale. Lecture Notes in Mathematics, vol. 569. Springer-Verlag, Berlin-New York (1977)

7. Gross, B.H., Reeder, M.: Arithmetic invariants of discrete Langlands parameters. Duke Math. J. 154(3), 431-508 (2010)

8. Hedayatzadeh, S. M. H.: Exterior powers of Barsotti-Tate groups, Ph.D. thesis, ETH Zürich, (2010)

9. Harris, M., Taylor, R.: The geometry and cohomology of some simple Shimura varieties, vol. 151 of Annals of Mathematics Studies, Princeton University Press, Princeton, NJ, with an appendix by Vladimir G. Berkovich (2001)

10. Huber, R.: A generalization of formal schemes and rigid analytic varieties. Math. Z. 217(4), 513-551 (1994)

11. Imai, N., Tsushima, T.: Geometric realization of the local Langlands correspondence for representations of conductor three, arXiv:1205.0734, to appear in Publ. Res. Inst. Math. Sci. (2012)

12. Imai, N., Tsushima, T.: Affinoids in the Lubin-Tate perfectoid space and simple supercuspidal representations I: tame case, arXiv:1308.1276, to appear in Int. Math. Res. Not. (2013)

13. Imai, N., Tsushima, T.: Local Galois representations of Swan conductor one, (2015), arXiv:1509.02960

14. Imai, N., Tsushima, T.: Affinoids in Lubin-Tate surfaces with exponential full level two, in Around Langlands correspondences, vol. 691 of Contemp. Math., pp. 157-180, Am. Math. Soc., Providence, RI, (2017)

15. Imai, N., Tsushima, T.: Stable models of Lubin-Tate curves with level three. Nagoya Math. J. 225, 100-151 (2017)

16. Imai, N., Tsushima, T.: Geometric construction of Heisenberg-Weil representations for finite unitary groups and Howe correspondences, (2018), arXiv:1812.10226

17. Imai, N., Tsushima, T.: Local Jacquet-Langlands correspondences for simple supercuspidal representations. Kyoto J. Math. 58(3), 623-638 (2018)

18. Jannsen, U.: Mixed motives and algebraic $K$-theory, vol. 1400 of Lecture Notes in Mathematics, Springer-Verlag, Berlin, with appendices by S. Bloch and C. Schoen (1990)

19. Laumon, G.: Transformation de Fourier, constantes d'équations fonctionnelles et conjecture de Weil, Inst. Hautes Études Sci. Publ. Math. (65):131-210 (1987)

20. Reeder, M., Yu, J.-K.: Epipelagic representations and invariant theory. J. Am. Math. Soc. 27(2), 437477 (2014)

21. Scholze, P.: Perfectoid spaces. Publ. Math. Inst. Hautes Études Sci. 116, 245-313 (2012)

22. Groupes de monodromie en géométrie algébrique. II, Lecture Notes in Mathematics, Vol. 340, SpringerVerlag, Berlin-New York: séminaire de Géométrie Algébrique du Bois-Marie 1967-1969 (SGA 7 II). Dirigé par P. Deligne et N. Katz (1973)

23. Scholze, P., Weinstein, J.: Moduli of p-divisible groups. Camb. J. Math. 1(2), 145-237 (2013)

24. Tokimoto, K.: Affinoids in the Lubin-Tate perfectoid space and special cases of the local Langlands correspondence. Math. Ann. 377(3-4), 1339-1425 (2020)

25. Weinstein, J.: Good reduction of affinoids on the Lubin-Tate tower. Doc. Math. 15, 981-1007 (2010)

26. Weinstein, J.: Semistable models for modular curves of arbitrary level. Invent. Math. 205(2), 459-526 (2016)

27. Yoshida, T.: On non-abelian Lubin-Tate theory via vanishing cycles, in Algebraic and arithmetic structures of moduli spaces (Sapporo 2007), vol. 58 of Adv. Stud. Pure Math., pp. 361-402, Math. Soc. Japan, Tokyo, (2010)

Publisher's Note Springer Nature remains neutral with regard to jurisdictional claims in published maps and institutional affiliations. 